http://dx.doi.org/10.12775/szhf.2016.013

\author{
Gerhard Preyer
}

Johann Wolfgang Goethe-Universität, Frankfurt am Main, Deutschland

\title{
Von der Radikalen Übersetzung zur Radikalen Interpretation - Quine, Davidson und darüber hinaus
}

\author{
Einleitung
}

Donald Davidson hat sich im Hinblick auf die Bedeutungstheorie natürlicher Sprachen grundsätzlichen Positionen der naturalisierten Erkenntnis-theorie Willard van Orman Quines angeschlossen ${ }^{1}$. Das gilt unabhängig von seiner Hinwendung zu einer distalen Bedeutungstheorie seit Anfang der 1980er Jahre als eine Kritik an der Reizbedeutung, der Zurückweisung der ontologischen Relativität und der Kritik am „Dritten Dogma des Empirismus“. Sein Umbau der radikalen Übersetzung (RÜ) zur radikalen Interpretation (RI) und ihrer wahrheitsbedingten Bedeutungstheorie überführt er in eine

${ }^{1}$ Die meisten seiner Artikel sind erschienen in [Davidson 1980, 1984, 2001, 2004, 2005]. Zu erwähnen ist von den nicht in diesen Bänden aufgenommenen Artikel vor allem [Davidson 1990, S. 279-328].

$\mathrm{Zu}$ Davidsons Philosophie, aus der wirklich mehr als reichhaltigen Sekundärliteratur seit der Mitte der 1975er Jahren, mit Gesamtüberblicken, z.B. [Lepore, Ludwig, 2005, 2007], [Ludwig 2003], zum neueren Forschungsstand [Preyer 2012a, 2012b], [Preyer, 2012b, S. 107-181], [Amoretti, Preyer 2013b], zu einer Anwendung auf die interpretative Soziologie [MacDonald, Pettit, 1981]. 
"Unified Theory of Thought, Meaning, Action and Evaluation“ [Davidson, 1980, S. 151-166]. Er teilt mit Quine die Annahme der postempiristischen Bedeutungstheorie, d.h. des Zirkels zwischen Überzeugung und Bedeutung, die Kritik an der semantischen Unterscheidung zwischen analytischen und synthetischen Sätzen, die Unerforschbarkeit der Referenz und die Unbestimmtheit der Übersetzung, den Extensionalismus in der Logik und eine Version der naturalisierten Erkenntnistheorie. Insofern sollten wir von einer Quine-Davidson-Tradition ausgehen.

In einem ersten Schritt wird die Ablösung der Bedeutungstheorie durch die Übersetzungstheorie skizziert (Quine). Das betrifft die Hinwendung zur naturalisierten Erkenntnistheorie, die postempiristische Bedeutungstheorie und die radikale Übersetzung (RÜ) sowie ihre Basistheorie ${ }^{2}$. Daran schließt sich die Kritik an, dass die Reizbedeutung als Nettobedeutung die epistemischen Anforderungen an die RÜ nicht klären kann (1. (a) - (d)). In dem zweiten Schritt gehe ich auf den Umbau der RÜ durch die radikale Interpretation (RI) von Davidson ein und skizziere ihre semantische Fassung der RÜ als eine Beibehaltung des Problembezugs der RÜ und zugleich der Zurückweisung ihrer Basistheorie (2. (a) - (c)).

Von Interesse ist es, ob Davidsons wahrheitsbedingte Bedeutungstheorie, somit die interne Beziehung zwischen Interpretation (Sprache) - Rationalität (Grundsatz der Nachsicht) - Externalismus, die epistemische Lücke in Quines Bedeutungs- als einer Übersetzungstheorie zu schließen vermag und ob die Basistheorie der RI den Zirkel zwischen Überzeugung (allen propositionalen Einstellungen) und Bedeutung erfolgreich unterbricht. Daran schließt sich das lehrreiche Folgeproblem an: Stellt die Wahrheitstheorie im Stile A. Tarskis eine zutreffende Antwort auf die Instanziierung des Wahrheitsprädikats bereit? (3.). Ein Ausblick auf einen alternativen Ansatz schließt den Durchgang durch die Anforderungen an die RÜ und RI ab (4.) $)^{3}$.

${ }^{2}$ Der Begriff der Basistheorie als Bezugsproblem des Vergleichs für die jeweils angenommene unbezweifelbare Grundlage der Erkenntnis, die den Zweifel begrenzt, geht auf [Schnädelbach, 1971, S. 13-14] zurück.

${ }^{3}$ Die in dem Text vertretene Position hat eine längere Vorgeschichte. 1980 konstituierte sich eine Gruppe, die sich mit den damals aktuellen Ansätzen von z.B. W. v. O. Quine, Davidson, V. Sellars, R. Rorty, H. Putnam, T. Burge und S. Kripke auseinandersetze. Rorty machte in seiner Vorlesung am Institut für Philosophie im Sommersemester 1975 Davidson unter den Seminaristen bekannt. Ein Ergebnis dieser Gruppe war, dass sich in der naturalisierten Erkenntnistheorie das Problem der epistemischen Anforderungen an die radikale Übersetzung und Interpretation stellte. Diese Problemstellung war durch Putnam, The Meaning of ,Meaning, [Putnam 1975, S. 215-271] Universalität der sozio-linguistischen Hypothesenbildung moti- 
Zugespitzt besagt mein Argument, dass das ,Selbstbewusstsein' der Quine-Davidson-Tradition in einem selbstreferenziellen ontologischen Fehlschluss besteht, da die eigene Ontologie und Logik, das gilt auch für R. Rorty, somit der gesamte sozio-strukturelle (kulturelle) Hintergrund, nicht nur vorausgesetzt, sondern als nicht zu hintergehen, behauptet wird. Wenn das dargestellte Argument zwingend sein sollte, dann bricht der tragende Stützpfeiler dieser Tradition und der naturalisierten Erkenntnis- und Sprachtheorie zusammen. Das betrifft nicht die kompositionale Semantik, sondern die verhaltenswissenschaftliche Grundlage der Sprach- und Bedeutungstheorie und ihre Rationalitätsstandards (Widerspruchfreiheit, Wahrheit, rationale Entscheidungstheorie), die nicht zu erneuern sind ${ }^{4}$.

Anmerken möchte ich noch zu der Vorgehensweise der Rekonstruktion von Basistheorien, dass sie den Vergleich unterschiedlicher Ansätze erleichtern. Der Wechsel in einer Basistheorie kann auch der Indikator dafür sein, dass ein Verlust an Plausibilisierungen vorliegt, z.B. äußert sich Davidson dahingehend, dass er Quines Begriff der Reizbedeutung nicht nachvollziehen kann. Wenn es sich dabei nicht nur um eine idiosynkratische Äußerung handelt, dann ist damit die reinterpretative Strategie des Interpreten im Hinblick

viert [Putnam 1975, S. 227-229], die eine epistemische Differenzierung von Experten in einer Sprachgemeinschaft beinhaltet. Insofern erfordert die intelligible Redeskription von sprachlichem und nicht-sprachlichem Verhalten der reinterpretativen Strategien eines Interpreten. Das ist eine Folgerung aus Burges „Arthritis-Gedankenexperiment“, [Burge (1979) 2007, S. 100-150]. M. Roth hat diese Problemstellung in seiner Promotion weiter verfolgt [Roth, 1986]. Im Fortgang wurde dieser Ansatz bei der Analyse in dem Sprechakttheorie-Projekt der ProtoSociology berücksichtigt - [Preyer, Ulkan, Ulfig 1996]. Freigeschaltet Academia unter Gerhard Preyer, Einheit: Action, Intention, Communication. Zur analytischen Handlungstheorie [Preyer 2011b].

${ }^{4}$ Historisch ist dazu anzumerken, dass N. Chomskys, Verbal Behaviour. [Chomsky 1959, S. 26-58] Kritik an B. F. Skinner, [Skinner, 1957] vom grundsätzlichen her gesehen, sie mag etwas unfair gewesen sein, zutreffend ist. Auch Quine, Replies, To Chomsky, [Quine, (1969) 1975, S. 302-311] auf Chomsky, Quine's Empirical Assumptions, [Chomsky, (1969) 1975, S. 53-68] war nicht so überzeugend. Es ist diesbezüglich zu erwähnen, dass der Naturalismus und seine pragmatische Ergänzung zwar eine dominierende Strömung der Philosophie seit den 1970er Jahren war, sie hat aber nicht alle Philosophen überzeugt. Dazu [Burge, (1993) 2007, S. 360]. Zu erwähnen ist auch der Gegenzug gegen die Kritik an der analytisch-synthetisch Unterscheidung und der verhaltenswissenschaftlichen Bedeutungstheorie von [Katz, (1966), 1969, S. 170-285].

Auf Quines „Method of Logik“ und seine Mengenlehre gehe ich nicht ein. Es ist jedoch anzumerken, dass Quine und N. Goodman ihren Nominalismus gegenüber ihren Kritikern nicht erfolgreich verteidigt haben; vgl. dazu [Stegmüller, 1978]. 
auf die Plausibilität von erkenntnistheoretischen Voraussetzungen angesprochen. Wie weit die damit einhergehenden erkenntnistheoretischen und ontologischen Umschichtungen gehen, ist eine empirische Frage.

\section{Bedeutungstheorie als Übersetzungstheorie}

\section{(a) Naturalisierte Erkenntnistheorie}

Aus den Texten von und aus den Gesprächen mit Philosophen kann man sich darüber informieren, das ist nicht weiter strittig, dass sich Ontologie und Erkenntnistheorie zunächst mit unterschiedlichen Fragen beschäftigen. Die Ontologie fragt danach, was es gibt. Es mag bei der Beantwortung dieser Frage „Limit myths“ (Quine) geben, z.B. infinitesimale Größen oder ideale Gegenstande, z.B. Massenpunkte, reibungslose Flächen oder isolierte Systeme Quine, 1979, S. 248-251]. Das ist eine Frage der Wahrheit. Die Erkenntnistheorie beschäftigt sich dagegen mit der Frage, wie wir wissen können, was es gibt. Das ist eine Frage der Methode (Verfahren) und der Belege ${ }^{5}$. Ontologie und Erkenntnistheorie sind jedoch andererseits nicht voneinander unabhängig, wenn wir aus der Sicht der Erkenntnistheorie nach ihren ontologischen Voraussetzungen fragen. Das betrifft in der Cartesianischen und empiristischen Tradition, z.B. das Subjekt-Objekt Problem oder die angenommene Beziehung zwischen Sprache (Logik) und Welt, z.B. den logischen Atomismus (B. Russell), das Konstitutionssystem des logischen Aufbaus der Welt (Rudolf Carnap), die starre Bezeichnung und metaphysischer Essentialismus (S. A. Kripke) oder die Annahme einer vorsprachliche Intentionalität, die physischen (phonetischen) Äußerungen eine Bedeutung verleiht (J. R. Searle: interne und externe Intentionalität).

Quines Erkenntnistheorie, Ontologie, Sprachtheorie und Logik beansprucht eine Antwort auf die Problemstellungen: 1. Die Beziehung zwischen Übersetzung und Kommunikation, 2. die Analyse der Referenz von Ausdrükken sowie 3. eine Analyse der Belege und des Wissens (Wissenschaftstheorie und Logik) zu geben. Das Verbindungsstück ist die naturalisierte Erkennt-

\footnotetext{
5 „Belege“ ist die übliche deutsche Übersetzung von „evidence“. Das ist nicht ganz glücklich, da „evidence“ bei Quine kein externer Beleg von etwas ist, sondern ein stimulierter Informationsfluss an den Nervenenden (flow of evidence), der nicht intentional bewusst ist. Das hängt wissenschaftstheoretisch bei Quine damit zusammen, dass die Überprüfung von Theorien in der „Vorhersage von Stimulationen“ besteht. In diesem Text bleibe ich der Einfachheit halber bei der verbreiteten deutschen Übersetzung.
} 
nistheorie. Die Kommunikation der Ergebnisse seiner Analysen beschreibt Quine, wie der Untertitel von Unterwegs zur Wahrheit lautet, als Konzise Einleitung in die theoretische Philosophie [Quine (1992) 1995].

Die naturalisierte Erkenntnistheorie besagt:

1. Es gibt keinen erkenntnistheoretischen ausgezeichneten Standpunkt der Erkenntniskonstitution und keinen ontologischen (kosmologischen) Standpunkt, z.B. den LaPlace-Dämon, von dem wir die äquivalenten Ontologien überblicken könnten. Das ist ihre Kritik an der Prima Philosophia. Der erkenntnistheoretische Standpunkt setzt jedoch den ontologischen voraus. Es gibt somit keine Erkenntnistheorie außerhalb der ontologischen Bindungen. Das gilt für unsere wissenschaftlichen Theorien und die logische Bereinigung der Alltagssprache. Nach Quine besagt das ontologische Kriterium: "to be is to be the value of the variable“. Damit wird die Quantifikation zweiter Stufe zurückgewiesen. Der ontologische Standard besagt: „no entity without identity“. Damit sollen intensionale Begriffe (Propositionen, Eigenschaften usw.) widerlegt werden. Das ist der logisch-semantische harte Kern der Erkenntnistheorie und Semantik Quines.

Aus dem ersten Punkt folgt, dass im Falle der Bedeutungstheorie als einer Übersetzungstheorie „Übersetzung" durch die nicht in Frage gestellten Hintergrundsprache des Übersetzers erfolgt (vgl. dazu den 4. Punkt).

2. Die Sprache und die Bedeutung von Ausdrücken sind keine privaten Entitäten (Musemus Mythos, Davidson: Mythos des Subjektiven). Aus der Perspektive der ersten Person haben wir keinen privilegierten $\mathrm{Zu}$ gang zu semantischen Informationen.

Die Rolle des Übersetzers besteht darin: (a) Er verfügt über eine begriffliche Souveränität, (b) die Übersetzung erfolgt von einem Begriffsschema und seiner Hintergrundontologie aus. (c) Daran schließt sich das Folgeproblem an, in welcher Position und wie das Interpretierte vom Standpunkt des Übersetzers (Interpreten) zu berücksichtigen ist?

3. In einem methodischen Sinn sind semantische Untersuchungen eine Sache der logischen Notation. Sie ist nicht theoretisch, da sie unser empirisches Wissen nicht erweitert. Sie ist aber auch nicht metaphysisch, da die Ontologie, wie alle Konzeptualisierungen, relativ zu einem Begriffsschema und deshalb nicht absolut ist. Quine neigt aber dazu, der kanonischen Notation eine große epistemische Nähe zu der Peripherie, somit den sensorischen Reizen, der begrifflichen Netzwerke zuzuschreiben. 
4. Es gibt keine selbstständige (philosophische) Erkenntnistheorie. Sie ist Aufgabe der Einzelwissenschaften, z.B. der Psychologie und Verhaltenswissenschaft.

Quine verbindet deshalb die naturalisierte Erkenntnistheorie mit der ontologischen Relativität, dem Dualismus zwischen neutralem Inhalt und Begriffsschema. Referenz wird durch die Relativierung auf ein Koordinatensystem erklärt. Dieses Koordinatensystem wird durch die Hintergrundsprache bereitgestellt. Die relative Referenz ist somit aus seiner Sicht ein akzeptabler semantischer Begriff, welcher der radikalen Übersetzung durch die Hintergrundsprache zur Verfügung gestellt wird. Die Sprache und die damit einhergehenden Ontologie des Übersetzers haben somit eine privilegierte Funktion.

Die relationale Theorie der Gegenstände ist der Kern der Lehre der ontologischen Relativität. Ihr kommt im Fortgang der Werkgeschichte Quine eine grundlegende Funktion in der Erkenntnistheorie und Ontologie zu [Quine (1969) 1971, S. 97-126; Quine 1995, S. 1-26; Quine 1992, S. 31-49]': „Aber ich kann heute vielleicht deutlicher als in meinen Vorlesungen, dem Essay oder dem Buch des gleichnamigen Titels („Ontologische Relativität“, d.V.) angeben, worauf die ontologische Relativität meiner Position zufolge eigentlich relativ bezogen ist: sie ist relativ auf ein besonderes Manual der Übersetzung. Vom Term „gavagai“ zu sagen, dass er Kaninschen bezeichnet, heißt nichts anderes, als sich anstelle irgendeines der anderen möglichen Handbücher für ein Handbuch zu entscheiden, in dem der Term "gavagai“ mit „Kaninchen“ übersetzt wird“ [Quine 1992, S. 73]. Die Nettobedeutung ist die Selektionsinstanz der „holophrastischen Ja-oder- Nein-Entscheidungen über die Beobachtungssätze, in die menschliche Prognosen eingehen“ [Quine 1992, S. 49]. Für die Basistheorie heißt das, dass die Basissituation der Erkenntnis und der Übersetzung nicht der „Term“, sondern der „monolitische Satz“ ist [Quine 1992, S. 49]. Damit geht einher, dass die Ontologien relativ sind und wir über keinen Standpunkt verfügen, über ihre Richtigkeit zu entscheiden. Der empirische Gehalt einer Ontologie ist nur der „neurale Knoten“ und ihr Beitrag zu der jeweiligen "Struktur der Theorie" [Quine 1992, S. 45]. Wir sollten uns dabei aber zugleich fragen, von welchem Standpunkt aus, diese Unterscheidung getroffen wird. Setzt die Unterscheidung nicht einen weiteren höheren

\footnotetext{
${ }^{6}$ Die Behauptung der ontologischen Relativität stützt sich auf das Permutationsargument und die Stellvertreterfunktionen vgl. dazu [Quine, (1969) 1971, S. 78-83], vgl. dazu „Gleichgültigkeit“ [Quine 1992, S. 42-45] und „Auflösung der Ontologie“, [Quine 1992, S. 45-49] zur „Unbestimmheit des Bezugs" [Quine 1992, S. 71-74].
} 
Standpunkt voraus? Wenn es keinen absoluten Standpunkt gibt, so sind die Sätze: „Es gibt einen absoluten Standpunkt“ und „Es gibt keinen absoluten Standpunkt" ohne einen Erkenntniswert. Die von Quine nahegelegte Unterscheidung lässt sich somit nicht absolut treffen ${ }^{7}$.

(b) Postempiristische Bedeutungstheorie und die radikale Übersetzung

Die postempiristische Bedeutungstheorie besagt: Die nicht auflösbare Verbindung zwischen Überzeugung und Bedeutung ist das Kernproblem der verständlichen Wiedergabe einer Äußerung (Sprechakt). Davidson formuliert dieses Kernproblem dahingehend: „A central source of trouble is the way beliefs and meaning conspire to account for utterance" [Davidson, (1974), 1984, S. 142]. Dieser Zirkel ist von der Anlage her nicht zu beseitigen (Nichtherausschälbarkeitsthese). Quine verwirft deshalb die analytischen Konzeption der sprachlichen Bedeutung (R. Carnap), den Dualismus von Bedeutungswahrheiten in einer gegebenen Sprache versus Tatsachenwahrheiten und die Unterscheidung zwischen internen und externen Fragen im Hinblick auf die sprachlichen Rahmenwerke (Carnap).

Der philosophische und sprachtheoretische Hintergrund ist Quines Kritik an der Bedeutungstheorie des logischen Empirismus und der damit einhergehenden „Zwei Dogmen des Empirismus“. Daraus folgert er: Weder Überzeugungen, noch Wahrheit, legen die Referenz von Ausdrücken fest. Seine Erkenntnistheorie ersetzt deshalb die phänomenologische Basis des Konstitutionssystems (Carnap) durch eine naturalistische Basis. Er bezeichnet seine Erkenntnistheorie auch als einen "relativen Empirismus" [Quine, (1974) S. 189-194; 1994, S. 191]. Er besagt: „Wage dich nicht weiter von den Sinnesdaten weg als nötig." Daran hat er werkgeschichtlich festgehalten [Quine, 1995, S. 1-26].

Die Basissituation des Gedankenexperiments der RÜ ${ }^{8}$ soll beides erklären, die Unerforschbarkeit der Referenz, d.h. die Unbestimmtheit von unten und

\footnotetext{
${ }^{7}$ Das ist die Folgerung aus einer Mailkommunikation über dieses Problem mit W. K. Essler.

${ }^{8}$ Quine, Word and Object (1960), zur radikalen Übersetzung S. 26-31. Der Aufbau des Buches ist derart organisiert, dass die erkenntnistheoretische Ausgangssituation in Kapt. I. dargestellt wird. Sie besteht darin, dass Begriffsbildung und Sprache immer zusammenspielen, ,wir' in der Begriffsbildung souverän sind und die Analyse von Theorien „in der Mitte“ anfängt, z.B. bei unserem Vorzug für „mittelgroße Dinge“. Zur wissenschaftlichen Methode gehört dabei, sich von Sinnenreizungen leiten zu lassen. Kapt. II. behandelt die „Nichtwahrnehmung der Unbestimmtheit“. Daraus folgt, dass die Annahme von Intensionen, durch die „Unbe-
} 
die Unbestimmtheit der Übersetzung, d.h. die Unbestimmtheit von oben. Die Situation der RÜ ist so definiert, dass z.B. ein Linguist (Feldforscher) die Äußerungen eines Sprecher einer Dschungelsprache - wir können verallgemeinern eine beliebige Sprache, die er nicht kennt - in seine Sprache von einem Nullpunkt aus zu übersetzen beginnt. Quine hält es im Fortgang für „,besser“, statt von Unerforschbarkeit von Unbestimmtheit des Bezugs zu sprechen.

Die Unbestimmtheit der Übersetzung von unten besagt:

1. Die Referenz als die gegenständliche Bedeutung von Ausdrücken ist aus der Einstellung der dritten Person unerforschbar. Referenz als eine Ausdrucks-Gegenstandbeziehung besagt: 1. als Designation eines singulären Terms, z.B. „Boston“ designiert Boston, 2. als Denotation eines generellen Terms „Hase“ denotiert jeden Hasen und 3. als der Wert von gebundenen Variablen [ Quine, 1995, S. 59-67].

Wenn ein Sprecher z.B. „Hase“ äußert, so kann die Referenz von einem radikalen Übersetzer bzw. Feldlinguist nicht verhaltensmäßig erforscht werden. Warum verhält sich das so? Das Problem ist durch die Individuation der Terme in der Situation der RÜ veranlasst. Es ist ein Problem auf der Seite des Übersetzers, da der Sprecher die Terme nicht individuiert. „Hase“ könnte z.B. ein Klassifikationsausdruck (allgemeiner Begriff) oder ein rein singulärer Term, z.B. ein raum-zeitlicher Teil des Hasen, sein. Es ist demnach sinnlos, absolut festzustellen, dass sich „Kaninchen“ auf Kaninchen oder auf abgetrennte Kaninchenteile und dass sich, relativ zu einer alternativen Menge, „Kaninchen“ auf unabgetrennte Kaninchenteile bezieht. Der Sprecher kann aber auch besondere Theorien über Hasen haben, z.B. sie sind Boten von fremden Göttern oder der Hase ist ein künstliches Replikat eines Hasen.

2. Die Übersetzung ist unbestimmt von oben, da die Wahrheit der Übersetzung eines Satzes am Leitfaden der naturalisierten Erkenntnistheorie nicht über seine Richtigkeit entscheidet. Es können immer andere analytischen Hypothesen aufgestellt werden. Die Unbestimmtheit der

stimmtheit“ zurückgewiesen wird. Kapt. III geht auf die „Ontogenese des Bezeichnens“ ein, systematisiert den Objektbezug und die „Setzung neuer Gegenstände“. Kapt. IV. beschreibt die Anomalien und Konflikte des Bezeichnens, die mit dem Hilfsmittel der modernen Logik einer Lösung zugeführt werden (Kapt. V., VI.). Kapt. VII. geht auf die „ontologischen Entscheidungen“ ein, und die allgemeine Anwendung des „semantische Aufstiegs“ dekonstruiert eine inhaltliche Ontologie.

${ }^{9}$ Quine, [Quine (1992), S. 71-74; Quine 1970, S. 179-183] hat beide Unbestimmtheiten im Fortgang genauer unterschieden; [Quine 1990, S. 1-16]. 
Übersetzung von oben als holophrastische These ist aus dieser Sicht die "stärkere" Unbestimmtheit, da sie nicht nur Terme, sondern auch ganze Sätze betrifft ${ }^{10}$.

3. Eine dritte Unbestimmtheit ist die empirische (Unter-) Unbestimmtheit der „Globalwissenschaft“. Das ist die Unbestimmtheit von Theorien durch die Beobachtungssätze, d.h. „Für den Fall von Globalsystemen der Welt hingegen sind wir sehr wohl zu akzeptieren bereit, dass es zahllose, nicht weiter spezifizierbare Möglichkeiten gibt, auf welche die Realität über die Grenzen des menschlichen kognitiven Apparats hinausreicht" [Quine, (1992), 141-142].

Der Übersetzer verfährt in der Einstellung der dritten Person, und er wendet den Grundsatz der Nachsicht, d.h. die zweiwertige Logik, bei der Übersetzung an, z.B. bei der Übersetzung der Junktoren. Es ist keine Anforderung an die Übersetzung, dass der Sprecher die Sätze kennt, in die der Übersetzer seine Äußerungen übersetzt. Der radikale Übersetzer stellt ein Übersetzungsmanual (analytische Hypothesen) auf. Die Übersetzung ist unter dieser Voraussetzung unbestimmt und die Referenz als eine Ausdrucks-Gegenstands-Beziehung ist nicht erforschbar, da es nicht zu entscheiden ist, welche analytischen Hypothesen definitiv richtig sind. Sie sind durch die Gesamtheit ihrer Belege unbestimmt. Die Referenz von Ausdrücken ist somit keine Tatsachenfrage, d.h. es gibt keine absolute Referenz, sondern Referenz ist relativ zu einer Rahmentheorie. In der Situation der RÜ sind deshalb die Unbestimmtheit der Übersetzung von unten (Unerforschbarkeit der Referenz) und die Unbestimmtheit der Übersetzung von oben, die zu unterscheiden sind, miteinander verbunden. Dieses Bild der Erkenntnistheorie folgt aus Quines postempiristischen Bedeutungstheorie: Alle Einstellungen wirken in eine für uns unauflösbare Weise zusammen und können nicht als einzelne Einstellungen isoliert werden.

Quine führt deshalb den Begriff der Reizbedeutung und der Reizsynonymie ein, mit der der radikale Übersetzer die Nettobedeutung aus dem Sprecherverhalten herausfiltert: "The stimulus meaning of a sentence for a subject sums up his disposition to assent to or dissent from the sentence in response to present stimulation. The stimulation is what activates the disposition, as opposed to what instills it (even though the stimulation chance to contribute somehow to the instilling of some further disposition)" [Quine 1979,

${ }^{10}$ Zur Kritik Quines von Davidson, H. Field und S. Leeds vgl. [Schantz, 1996, S. 196-216]. Schantz hat das grundlegende Problem von Quines Ansatz aus meiner Sicht gut identifiziert. 
S. 34; zur Stimulation und Reizbedeutung S. 31-35]. Die Reizbedeutung ist zugleich das Verbindungsstück zwischen Erkenntnistheorie und Ontologie: „The stimulation of his sensory receptors is all the evidence anybody has had to go on, ultimately, in arriving at his picture of the world" [Quine, (1969), S. 75]. Die sensorischen Reizungen sind aus dieser Sicht die Ursachen, d.h. die natürliche Basis, unserer wissenschaftlichen Theorien über die Welt. Diese Reizbedeutung stimmt jedoch nicht mit unserem intuitiven (normalen) Bedeutungsverstehen überein.

Die Beobachtungen sind nach Quine nicht etwas Sprachliches. Sie sind nur durch die Beobachtungssätze (Gelegenheitssätze), z.B. „Dies ist rot“ und „Das ist ein Hund“, intersubjektiv zugänglich. Sie sind „stimulus analytisch", d.h. sie beziehen sich auf bestimmte Situatitonen und werden durch "gleiche Reize“ aufgelöst. Er greift nicht auf Sinnesdaten zurück, sondern auf gemeinsame Reaktionen, d.h. wir können uns auf diese "Sätze“ einigen, und sie "sind das Eingangstor zur Sprache und zur Wissenschaft"11. Nach Quine ist die Basis der Übersetzung dieselbe Reizbedeutung aufgrund gleicher Nervenreizungen, welche die Übereinstimmung der „Dschungelsprache" mit den Beobachtungssätzen der eigenen Sprache gewährleisten soll ${ }^{12}$.

Der Übersetzer wendet den Grundsatz der Nachsicht gegenüber dem Sprecher (Informant) an ${ }^{13}$, d.h.

1. Die Maximierung der Übereinstimmung zwischen Sprecher und Interpret, somit die Ausschaltung von Irrtümern,

\footnotetext{
${ }^{11}$ Quine, [1974, S. 65, 61-67], seine Analyse von kategorischen Beobachtungssätzen hat er werkgeschichtlich variiert. Zur Endfassung [Quine 1995, S. 12-16]. Ein kategorischer Beobachtungssatz ist ein aus Beobachtungsgliedern zusammengesetzter Satz derart: „Sobald dies, dann auch das“. Das „sobald“ ist nicht zeitlich zu lesen. Es beschreibt einen mit den beiden Beobachtungssätzen konstant einhergehenden Umstand, [Quine 1995, S. 13]. Es fällt dabei auf, dass diese Version indexikalischen Ausdrücken keinen Platz einräumt.

${ }^{12}$ Das Problem der Geltung der Reizbedeutung (Identität der Reizbedeutung) stellte sich werkgeschichtlich im Hinblick auf den individuellen Sprecher, eine Sprachgemeinschaft oder eine besondere Gruppe. Vgl. dazu zur Übersicht [Schantz, 1996, S. 126-131]. Es könnte z.B. sein, dass ein Beobachtungssatz für die Mitglieder einer Sprachgemeinschaft gilt, obwohl sie nicht über die Sprecherdisposition verfügen, diesem Satz durch seine Auslösung in der entsprechenden Situation zuzustimmen. Das Problem betrifft den empirischen Gehalt eines prüfbaren Satzes, vgl. [Quine, (1992), S. 22-25].

${ }^{13}$ Quine nimmt ein Motiv von N. L. Wilson auf.
} 
2. Der Selbstkonsistenz und die Übereinstimmung verschiedener Sprecher ist eine Voraussetzung der Zuordnung der Äußerung von Sätzen zu den Bedingungen der Äußerungssituation ${ }^{14}$.

3. Der radikale Übersetzer geht davon aus, dass die Einstellungen des Sprechers den seinigen ähnlich sind, und er unterstellt ihm seine eigene Ontologie. Das gilt solange, wie nicht das Gegenteil offensichtlich ist. Daraus folgt:

Die Wahrheit von Sätzen als Bezugsproblem der nicht-analytischen Bedeutung ist demnach eine einfache Korrespondenz von Sätzen und physikalischen Tatsachen in bestimmten Äußerungssituationen. Insofern sind privilegierte Klassen von Sätzen aufzugeben, wie z.B. analytische und synthetische Sätze.

4. Der radikale Übersetzer kann auch seine eigene Hintergrundsprache ins Wanken bringen, da die RÜ auch auf sie anwendbar ist. Das wird er aber nicht tun, da er sich dadurch sozusagen den „Boden unter den Füßen“ wegziehen würde [Quine, (1992), S. 68-69].

Quine erkennt letztlich keinen Unterschied zwischen der Unerforschbarkeit der Referenz und der ontologischen Relativität, da die Referenz der Terme einer Sprache ihre Ontologie bestimmt und die Relativität der Ontologie unmittelbar aus der Relativität der Referenz auf ein Begriffsschema (Sprache) folgt.

\section{(c) Basistheorie der RÜ}

Die Skizze soll soweit informativ sein, um die Basistheorie der RÜ zu verdeutlichen:

1. Die Bedeutung des intendierten Gehalts ist aus einer beliebigen Sprecheräußerung nicht herausschälbar.

\footnotetext{
${ }^{14}$ Davidson hat die Leitlinie der Maximierung durch die Optimierung der Übereinstimmung ersetzt, da es keinen Sinn hat, zwei unendliche Mengen von Sätzen gegenseitig zu maximieren. Eine Strategie war, das Problem dadurch zu entdramatisieren, dass dem Grundsatz der Nachsicht der Grundsatz der Humanität, dass der Sprecher nach Wahrheit strebt, vorgeschaltet wird, z.B. R. Grandy, Reference, Meaning and Belief, Journal of Philosophy Vol. LXX, No. 14. Die Strategie geht dahin, dass nicht vorrangig die Übereinstimmung maximiert, sondern die unverständliche Nichtübereinstimmung minimalisiert wird. Vgl. dazu auch MacDonald, Pettit, Semantics and Social Science, S. 29-38.
} 
2. Das Verstehen von Sätzen ist nicht „referenziell schematisierbar“: Überzeugung (Zusatzinformation) und Bedeutung sind untrennbar verbunden.

3. Nur Sätze einer Theorie und nicht Ausdrücke beziehen sich auf die Welt.

4. Die Fragen nach der Bedeutung von Sätzen sind als Fragen der Übersetzung in einer Rahmentheorie zu systematisieren.

Insofern ist das Bedeutungsverstehen auf die empirischen Belege einer Rahmentheorie zu spezifizieren. Für die Bedeutungstheorie natürlicher Sprachen wird dies an der Situation der radikalen Übersetzung exemplifiziert.

5. Die Nettobedeutung von Beobachtungssätzen wird an den Äußerungsbedingungen durch die Reiz-Reaktions-Beschreibung festgestellt.

Als wissenschaftstheoretische Hintergrundtheorien sind die Physik und die verhaltenswissenschaftliche Psychologie verbindlich.

6. Die Anwendung des Grundsatzes der Nachsicht (klassische Logik) gewährleistet die Vereindeutigung der nicht zu beseitigenden Unbestimmtheit der Übersetzung von oben, z.B. der logischen Junktoren.

7. Die relative Referenz ist somit ein akzeptabler semantischer Begriff, der dem radikalen Übersetzer durch seine jeweilige Hintergrundsprache zur Verfügung gestellt wird, z.B. deutsch, französisch, chinesisch, was auch immer. Die relationale Theorie der Gegenstände ist der Kern der Lehre der ontologischen Relativität.

Die Folgerung ist: Die Unbestimmtheit der Übersetzung belehrt uns darüber, dass es keine Propositionen als Satzbedeutungen gibt, und die Unterbestimmtheit der globalen Wissenschaft belehrt uns darüber, dass es nicht nur eine einzige Version des Denkens über die Welt gibt.

\section{(d) Einwände}

Das grundlegende Problem von Quines Ansatz ist: Gibt er auf die Problemstellungen 1. der Beziehung zwischen Übersetzung und Kommunikation, 2. der Referenz von Ausdrücken, 3. der Analyse der Belege und des Wissens sowie 4. der Annahme der Geltung der Verhaltenswissenschaft als Basistheorie der empirischen Psychologie eine tragfähige Antwort, die weiter innovierbar ist? ${ }^{15}$ Die Problemstellungen und die Fragen nach der Inno-

${ }^{15}$ T. Burge [2010, S. 216-288] hat diese Fragestellung im Hinblick auf Quine, Evans, Strawson und Davidson analysiert. Mit Burge stimme ich in vielen Punkten überein, z.B. dass diese 
vierbarkeit betreffen auch den Ansatz von Davidson. Angesprochen ist damit die Frage nach dem Verbindungsstück des Ansatzes: Wie passen die Thesen der Unerforschbarkeit der Referenz (Unbestimmtheit von unten), der Unbestimmtheit der Übersetzung von oben, die Relativität der Ontologie und dass es auf die Struktur einer Theorie ankommt, nicht auf die Wahl der Gegenstände, mit seinem „robusten Realismus“, dass es materielle Gegenstände in Raum und Zeit gibt und dem wissenschaftlichen Realismus zusammen?

1. Quines Erkenntnisinteresse ist die Klärung ontologischer Fragen. Das setzt er voraus. Insofern thematisiert er fremde Sprachen als gegebenenfalls alternative Theorien. Zwischen ihnen ist im Hinblick auf die Schema-Inhalt-Unterscheidung eine Vergleichbarkeit herzustellen.

Es ist an dieser Stelle im Blick zu behalten, dass die Annahme der Überzeugung als eine Verhaltensdispositon ihre semantisch signifikante Eigenschaften durch natürliche Eigenschaften ersetzt.

In der naturalisierten Erkenntnistheorie wird die Reizbedeutung dazu herangezogen, die im Definiens des Beobachtungssatzes auftretenden Begriff zu „objektivieren“, d.h. der untheoretischen Beobachtung zugänglich zu machen.

2. Wir erkennen an dieser Basistheorie der radikalen Übersetzung:

(a) Das Problem der Selektion der linguistisch relevanten Daten im Hinblick auf die Überzeugungen des Sprechers und

(b) es stellt sich die Frage, ob die Quine-Beobachtungssätze auch wirklich auf diese Situation anzuwenden sind?

Es liegt nahe, dass die Übersetzung der Satzäußerung des Fremden mehr erfordert, als die Überzeugung, was in der gegebenen Situation der Fall ist und durch die verhaltenswissenschaftliche Auswahl festgestellt wird.

3. Es betrifft dies die Problematik der Abgrenzung der Überzeugung von der Bedeutung. Es kann z.B. der Fall sein, dass der Einheimische und

Tradition keine angemessene Fassung der Perzeption hat und sich nicht im Einklang mit der empirischen Psychologie und Biologie befinden, z.B. die Ergebnisse der Forschungen über visuelle Systeme, sowie erkenntnis- und sprachtheoretisch nicht fortführbar ist, z.B. die "geteilte Referenz" der Unterscheindung in individuierte Einzeldinge (particulars) - vor allem Körper - und allgemeinen Typen. Es ist auch fraglich, ob die Quantifikation das allgemeine Prinzip der Identität und der Individuierung formuliert [Burge, 2010, S. 238-250]. 
der Feldforscher in der Bedeutung (Referenz) des Ausdrucks „Hase“ übereinstimmen, aber im Hinblick auf ein unterschiedliches Weltbild von dem beobachteten Hasen eine andere Interpretation vornehmen. Angesprochen ist damit die reinterpretative Strategie des Übersetzers.

Daraus ist zu folgern:

Die Bedeutung in Termini einer untheoretischen Bedeutung als erkenntnistheoretische Unterscheidung eines schemaunabhängigen Inhalts (sensory imput, stimulus meaning) von konzeptuellen Schemata erfasst gerade nicht den Hintergrund des Sprechers.

4. Die Spezifikation der Relativität der Satzbedeutung auf Physik und Verhaltenswissenschaft als Leitwissenschaft ist selbst nicht nachgewiesen, sondern wird als plausibel vorausgesetzt:

(a) Die Annahme der Satzwahrheit als eine einfache Korrespondenz von Satz und physikalischer Tatsache ist eine A priori-Annahme, die nicht verhaltenswissenschaftlich überprüft werden kann.

(b) Es gibt gerade keine offensichtliche Bedeutung, die an den Sprecheräußerungen zu beobachten ist bzw. die durch Reiz-Reaktionen ausgelöst wird.

Insofern trägt die Reizbedeutung nichts zur Unterbrechung des Zirkels zwischen Überzeugung und Bedeutung bei.

5. Daraus ist zu folgern, dass die offensichtliche Bedeutung und die epistemische unbeschränkte physikalische Wahrheit den epistemische Bestandteil des Sprechakts (Hintergrund) nicht erfassen kann, da der Zusammenhang zwischen Sprecherwissen und Bedeutung den Zirkel zwischen Überzeugung und Bedeutung schließt. Das ist der Problembezug der epistemischen Anforderungen und epistemischen Modifikationen, die beide betreffen, den Sprecher und den Übersetzer. Nehmen wir das ernst, so stellt sich die Frage, ob die postempiristische Bedeutungstheorie die richtige Fragestellung hat.

\section{Von der RÜ zur RI}

(a) Flucht vor der Reizbedeutung

Das Gesamtbild des philosophischen Ansatzes Davidsons, der sich in seinen Artikeln seit Anfang der 1960er Jahren dokumentiert, ist gut erforscht. 
Ihr übergreifender Problembezug ist durch die Problemstellungen der Erkenntnistheorie, Ontologie, Sprachtheorie und Logik Quines motiviert. Davidsons „Vereinheitlichte Theorie der Gedanken, Bedeutung und Handlung“ (als auch der „Bewertung“) beansprucht, die Beziehung zwischen Interpretation - Rationalität (Grundsatz der Nachsicht) - Externalismus mit sprachtheoretischen Mitteln zu erhellen und das Kapitel des Cartesanismus und Empirismus in der Philosophie zu schließen. Die Semantik einer Sprache ist aus dieser Sicht für ihre Ontologie erforderlich: „It is one thing to say that the semantics of a language forces us to engage with the ontology of the language; but can we learn anything from this about ontology itself, about what there is? I think we can. If we have the semantics of a language right, the objects we assign to the expressions of the language must exist. The proper semantic method leads to metaphysical conclusions" [Davidson, 2005, S. 40].

Von Interesse ist für mich sein Schritt zu der RI, seine Wahrheitsbedingungssemantik und die Basistheorie der RI. Daran anschließend wird die Korrektur des Ansatzes umrissen.

1. Die Methodologie der RI geht auf die Beschreibung der RÜ zurück ${ }^{16}$. Beide Ansätze gehen davon aus, dass die Belege des Sprachverstehens öffentlich zugänglich und damit offensichtlich sind. Die Explananda der Interpretationstheorie der RI sind „perceptual beliefs, the beliefs that are directly caused by what we see and hear and otherwise sense" [Davidson, 1999, S. 18-19] und die „sprachliche Kommunikation“ von individuellen Sprechern. Eine gemeinsame Sprache besteht demzufolge in der Übereinstimmung ihrer Interpretationstheorien. Insofern ist die RÜ und die RI durch beobachtbares Verhalten vollständig bestimmt. Alle Belege für oder gegen eine Übersetzung bzw. Interpretation sind somit durch die Ereignisse (Situationen) verursacht, denen der Sprecher zustimmt oder die er ablehnt. Aus dem beobachtbaren Verhalten können somit bestimmte Einstellungen der Sprecher zu Sätzen erschlossen werden.

2. Der radikale Interpret verfährt nicht, so wie im Fall der RÜ, mit einem verhaltenswissenschaftlichen Test, sondern er geht von einem mentalen

${ }^{16}$ Das ist von den textuellen Belegen nicht zu bezweifeln [Davidson, (1974), 1984, S. 141-154]: "We interpret a bit of linguistic behaviour when we say what a speaker's words mean on an occasion of use. The task may be seen as one of redescription. We know that the words 'Es schneit' have been uttered on a particular occasion and we want to redescribe this uttering as an act of saying that it is snowing" Davidson, (1974), 1984, S. 141]. 
(intensionalen) Begriff des Für-wahr-haltens aus, um die primären Daten der RI zu charakterisieren. Insofern ist seine Aufgabe von Beginn an eine semantische. Der einzige Begriff des Belegs, den Davidson akzeptiert, ist die logische Beziehung zwischen Überzeugungen und Sätzen, die sie ausdrücken. Der Beleg des Für-wahr-halten entscheidet dann darüber, dass die Sprache nicht mehr frei gewählt werden kann. Es ist aus der Sicht der RI unter dieser Voraussetzung eine empirische Frage, welche Sprache ein Sprecher spricht [Davidson, (1979), S. 240-241] ${ }^{17}$. Das schließt eine unvereinbare Interpretation der verfügbaren Belege nicht aus.

3. Die RI geht aber noch einen Schritt weiter. Nach ihr gibt es keinen Weg, von Verhaltenstatsachen zu semantischen Tatsachen, der nicht durch die Zuschreibung basaler propositionaler Einstellungen vermittelt ist. Insofern lehnt sie die Reizbedeutung ab. Davidson interpretiert sogar Quines Begriff der Reizbedeutung als einen Cartesianismus ${ }^{18}$. Eine semantische Theorie baut auf nichtsemantischen und nichtlinguistischen, jedoch auf keiner reinen Verhaltensbelegbasis auf. Die Sprachäußerungen sind zwar ein nichtsprachliches Handeln und ein physisches Ereignis, aber es wird einer begrifflichen und nomologischen Reduktion des Mentalen auf Physisches eine Absage erteilt. Deshalb ist der Begriff der Zustimmung und Ablehnung von Sätzen ein mentaler Begriff. Die Rede von „einen Satz für wahr halten“ beansprucht, dass es sich um eine Meinung (Überzeugung) handelt, die besagt, davon überzeugt zu sein, dass ein Satz wahr ist. Die Unabhängigkeit der Verfügbarkeit der Belege der RI soll damit gewährleistet sein.

4. Ein weiterer grundlegender Unterschied zwischen der RÜ und der RI ist es, worin die Ursachen bestehen, die den Inhalt dessen bestimmen, was wir sagen. RÜ und RI stimmen darin überein, dass die Belege für eine Interpretation in den Ereignissen bestehen, die kausal dafür ver-

${ }^{17} \mathrm{Zu}$ der Reinterpretation der Unbestimmtheit [Lepore, Ludwig, 2005, S. 221-242, Zu der Abstufung der Interpretationsebenen [Lepore, Ludwig, 2005, S. 228-229].

${ }^{18}$ Die proximale Bedeutungstheorie ist nach Davidson deshalb ein Cartesianismus, da sie von der Perspektive der ersten Person ausgeht und den Bereich des Subjektiven als Fundament der empirischen Erkenntnis einstuft. Dem cartesianischen Aufbau der Erkenntnis zufolge erfordert der Erwerb von Wissen über die Welt deshalb des erkenntnistheoretischen Schritt vom Bereich des Subjektiven zum Objektiven. Nach Davidson bleibt Quine dieser Erkenntnistheorie verhaftet. 
antwortlich sind, dass Sprecher bestimmten Sätzen zustimmen oder sie ablehnen.

Im Unterschied zur RÜ sind es nicht die Stimulationsmuster der sinnlichen Rezeptoren, die einen Sprecher dazu veranlassen, z.B. „Gavagai“ zuzustimmen, sondern die Beschreibung von äußeren (distalen) Ereignissen (Situationen), z.B. des Herumhüpfens von Kaninchen, was auch immer. Insofern vertritt Davidson einen ontologischen Realismus der Individuierung der Gegenstände (Inhalte) der propositionalen Einstellungen: Die Realität verbürgt ihre Wahrheit (distale Individuation der Inhalte propositionaler Einstellungen $)^{19}$. Die ontologische Relativität und der Dualismus zwischen „Inhalt und Schema“ wird als das „Dritte Dogma“ des Empirismus eingestuft und verworfen.

Davidson wendet gegen Quine ein, dass er nicht danach gefragt hat, worin die sensorischen Belege bestehen, auf denen unsere Überzeugungen über die Welt beruhen. Er wendet gegen die Quine-Belege der Stimulationen der sensorischen Rezeptoren ein, dass wir unter normalen Bedingungen über das, was sich an unseren Nervenenden abspielt, keine Kenntnisse haben. Die Ereignisse, die zu einer Zustimmung oder Ablehnung von Gelegenheitssätzen führen, sind entdeckbare Veränderungen in der Welt. Der Begriff der Reizbedeutung spielt für die RI keine Rolle.

5. Insgesamt wird dem radikalen Interpreten in seiner Ausstattung nicht nur eine prominente, sondern auch eine dominante Rolle zugesprochen. Quine ging von unserer begrifflichen Souveränität aus. Dem radikale Interpret wird eine neue Stellung zugeschrieben, da er ein „allwissender Interpret" ist, der das eigene Verhalten und die eigenen Wahrheitsmaßstäbe auf den Sprecher überträgt. Das Argument besagt: „We do not need to be omniscient to interpret, but there is nothing absurd in the idea of an omniscient interpreter; he attributes beliefs to others, and

${ }^{19}$ Davidson akzeptiert aber nicht H. Putnam's „radically non-epistemic“ Fassung der Wahrheit (Putnam: transcendental realism) und M. Dummetts „evidence-transcendent“ Realismus [Davidson, 1990, S. 298-299]. Er argumentiert demgegenüber [Davidson, 1999, S. 104-115]: "Correspondence, while it is empty as a definition, does capture the thought that truth depends on how the world is, and this should be enough to discredit most epistemic and pragmatic theories. Epistemic and pragmatic theories, on the other hand, have the merit of relating the concept of truth to human concerns, like language, belief, thought and intentional action, and it is these connection which make truth the key to how mind apprehends the world", [Davidson, 1999, S. 114]. Der Begriff der „objektiven Wahrheit“ soll das gewährleisten. 
interprets their speech on the basis of his own beliefs, just of the rest of us do" [Davidson, (1977), S. 201] ${ }^{20}$. Ein allwissender Interpret ist nach Davidson ein Interpret, der eine vollständige Kenntnis der Welt hat, ausgenommen der Kenntnis der Einstellungen von Sprecher und der Bedeutung ihrer geäußerten Sätze. Hinter den als global behaupteten Rationalitätsstandard kann der radikale Interpret gerade epistemisch nicht zurückgehen. Insofern ist der Grundsatz der Nachsicht ein „konstitutiver" Grundsatz („basic principle of rationality“). Irrationalität ist deshalb „innere Widersprüchlichkeit“ [Davidson (1982), S. 169-187; Davidson (1985), S. 189-198]. Nehmen wir diesen Standpunkt ein, so stellt sich die Frage: Hat jemand von diesem Standpunkt aus, einen Regenzauber, verstanden'?.

\section{(b) Wahrheitsbedingungssemantik}

1. Der Übergang von der RÜ zur RI besteht darin, dass die RI die Form einer Wahrheitstheorie im Stile Tarskis annehmen soll. ${ }^{21}$ Die Gegenstände der interpretativen Wahrheitstheorie für eine Sprache $x$ sind Äußerungen und Inskriptionen. Eine Wahrheitstheorie für eine Sprache $x$ ist eine finite und kompositionale Theorie, die über die formalen Mittel verfügt für jeden Satz der Sprache $x$ ein W-Theorem der Form „(W) s ist wahr $g d w p^{\text {“ }} \mathrm{zu}$ beweisen. ${ }^{22}$ Die Theoreme sind interpretativ, wenn

${ }^{20}$ Zur Kritik an der Begründung der Annahme des allwissenden Interpreten [Lepore, Ludwig, 2005, S. 323-329].

${ }^{21}$ Nach Tarski ist Semantik ein Fach, das die bestimmte Beziehung zwischen Ausdrücken einer Sprache und ihren Bezugsgegenständen untersucht. Er unterscheidet die semantischen Begriffe 1. das Benennen, die Referenz singulärer Termini, 2. das Erfüllen, die Beziehung eines offenen Satzes zu der Entität für die er steht und 3. das Definieren, die Beziehung zwischen einer Gleichung und der durch sie eindeutig bestimmten Zahl. Im Unterschied dazu bezeichnet das Prädikat „wahr“ keine Beziehung, sondern die Eigenschaft einer Klasse von bestimmten Ausdrücken, den Sätzen. Damit ist das Folgeproblem angesprochen, ob wir Propositionen als Wahrheitsträger in der Semantik und der Wahrheitstheorie als Wahrheitsbegriff benötigen.

${ }^{22}$ Kompositionalität besagt: Eine natürliche Sprache ist in primitive und komplexe Ausdrükke zu zerlegen. Ihre Relation besteht darin, dass die komplexen durch die Anordnung der primitiven Ausdrücke gebildet werden. „Ausdrücke“ sind abstrahiert (Abstraktoren), die unabhängig von ihrer Exemplifizierung bestehen. Das unterscheidet sie von akustischen und zwei-dimensonalen geometrischen Formen, dazu [Davidson, 2001, S. 107-108] im Hinblick auf den abstrakten Charakter der Sprache [Davidson, 2001, S. 108] und ihre Rolle, nicht für das Lernen der Muttersprache, sondern für die Erklärung des Sprachverhaltens von Philosophen, Psycholinguisten und Linguisten [Davidson, 2001, S. 108]. 
"p“ auf der rechten Seite der „Form W“ den Satz $s$ auf der linken Seite übersetzt. Die W-Theorie ist interpretativ, wenn sie nur interpretative W-Theoreme enthält.

Die analytischen Hypothesen des radikalen Übersetzers sind für den radikalen Interpreten in die Form von W-Sätzen z.B. „,,Schnee ist weiß“ $g d w$ Schnee weiß ist" umzuwandeln, die auf Sprecher und Zeitpunkte zu relativieren sind. ${ }^{23}$ Das wird „Davidson Konvention W“ (Ernest Lepore, Kirk Ludwig) genannt [Lepore, Ludwig, 2007, S. 41-42]. Die W-Sätze beanspruchen für die unendliche Menge von Sätzen der zu interpretierenden Sprache die formalen Anforderungen des Verstehens ihrer Bedeutung anzugeben. Tarskis Konvention $W$, angewandt auf natürliche Sprachen, erfordert es, dass die Theorie als Theoreme Instanzen des $W$-Schemas hat (,s ist $\left.W g d w p^{\prime}\right)$, in welchen $s$ durch den Satz , $p^{\prime}$ in der Metasprache übersetzt wird, den wir dazu verwenden, die Wahrheitsbedingungen der Satzäußerung eines beliebigen Sprechers anzugeben. Sofern wir ein Theorem identifizieren, das auf die Wahrheitsbedingung anwendbar ist, so ist, $s$ ist $W g d w p^{\prime}$ durch ,bedeutet dass' austauschbar. Wir sollten den Anspruch der interpretativen Wahrheitstheorie im Blick haben, um zu erkennen, dass nach diesem Ansatz Wahrheit nicht nur ein formaler, sondern ein explanatorischer Begriff ist, der gerade kein propositionales Wissen voraussetzt: ,... a theory of truth is a theory for describing, explaining, understanding, and predicting a basic aspect of verbal behaviour. Since the concept of truth is central to the theory, we are justified in saying truth is a crucially important explanatory concept" [Davidson, 1990, S. 313]. Dabei ist die besondere Rolle der W-Sätze im Blick zu behalten: Durch welche Operation sie auch immer hergestellt werden und welche Ontologie dabei behilflich sein mag, das Ergebnis ist, dass die Wahrheitsbedingungen der W-Sätze auf, eigenen Beinen'stehen.

Davidsons Umwandlung der RÜ in die RI besagt somit:

(a) Eine Theorie der Bedeutung stellt formale und empirische Anforderungen an die Wahrheitstheorie.

(i) Die formale Anforderung ist: Die Bikonditionalen der W-Sätze haben sowohl wahr als auch gesetzesartig zu sein, und sie sind aus einer endli-

${ }^{23}$ Davidson unterstellt dabei, dass die W-Sätze die von Tarski angenommene materiale Adäquatheitsbedingung erfüllen. 
chen Menge von Axiomen abzuleiten. Die W-Sätze sollen mehr leisten als eine Übersetzung.

Davidson hat keine genaue Parallele der W-Konvention für kontextsensitive Sprachen ( $=$ mit indexikalischen Ausdrücken) formuliert [Lepore, Ludwig, 2005, S. 47; Zu einem Vorschlag, S. 47-48]. Der „Davidson Konvention W-Satz" hat die ungefähre Form:

(W) „Gavagai“ (Da ist ein Gavagai) ist wahr in $L$, für einen Sprecher $x$ zum Zeitpunkt $t, g d w$ sich in der näheren Umgebung von $x$ zu $t$ ein Kaninchen befindet. Das gilt unter ceteris paribus Bedingungen. ${ }^{24}$

Es werden semantische Prädikate, bezogen auf Sprecher und Zeitpunkte, eingeführt (,bedeutet $(S, t)$, , ist wahr $(S, t)^{\prime}, \quad \operatorname{Ref}(x, S, t)$,' Ref=Referenz), um die relevanten Kontexte der Satzäußerungen zu interpretieren. Eine dazu parallele Anforderung an die Theoreme ist es, dass die Wahrheitstheorie unter ihren Theoremen alle Sätze der Form ,Für jedes $S, t$, $s$ ist wahr $(S, t) g d w p^{\prime}$ enthält, welche den $B$-Sätzen ,Für jedes $S, t, s$ bedeutet $(S, t) g d w p$ ) ist wahr' entsprechen. Die theoretische Anforderung besteht darin, dass die Axiome der Wahrheitstheorie für eine kontext-insensitive Sprache mit der Davidson Konvention $W$ als einer kontext-sensitiven Spezifikation auf Sprecher und Zeitpunkte zusammengeführt werden.

Die induktiv generalisierten Gelegenheitssätze haben die Form

“(L) For all speakers $S$, times $t$, ceteris paribus, $S$ hold true $s$ at $t$ iff $p$.

Where ' $S$ ' ranges over members of the target linguistic community, ' $p$ ' is replaced by an open sentence that specifies relative to time, and

${ }^{24}$ [Lepore, Ludwig, 2005, S. 195, 232-233]. Zu Rolle der ceteris paribus Klausel [Lepore, Ludwig, 2005, S. 190], [Ludwig, 1999, S. 37, 39]. Die ceteris paribus Bedingung wird durch den erklärenden Anspruch der wahrheitszentrierten Bedeutungstheorie und ihre Stützung durch die a priori Anwendung des Nachsichtsgrundsatzes als Brückengrundsatz der Adäquatheitsbedingung der empirischen Theorie der Wahrheit und Bedeutung erzwungen, da die Beobachtungssätze durch die kausale Auslösung der Wahrnehmungsüberzeugungen über die Umwelt des Sprechers und des Interpreten als für wahr einzustufen sind (Korrespondenzgrundsatz). Es ist jedoch empirisch nicht auszuschließen, dass einige dieser Überzeugungen falsch sind, z.B. der Hase, der ein Replikat ist. Insofern hat auch der Korrespondenzgrundsatz, das würde Davidson zugestehen, dem Irrtum einen Platz einzuräumen. 
perhaps other contextual features, conditions under which speakers hold the sentence true, and ' $s$ ' denotes an occasion sentence" [Lepore, Ludwig, 2005, S. 183]. Diese Sätze lassen jedoch die Lücke zwischen dem Für-wahr-halten und dem Wahrsein offen. Sie wird durch die Sätze der Form (TF), die „(L)“ nicht enthalten,

(TF) For all speakers $S$, times $t, s$ is true for $S$ at $t$ iff $p$

Geschlossen [Lepore, Ludwig, 2005, S. 184]. Das verweist auf den Grundsatz der Nachsicht „ceteris paribus (die meisten) Überzeugungen des Sprechers über seine Umwelt sind wahr" und seine unbegrenzte Anwendung zurück, der dafür die Gewährleistung übernimmt. Die Gewährleistung ist dadurch in Kraft gesetzt, dass die Sprecher "Sprecher“ sind. Das soll ohne irgendeine Annahme über ihre Gedanken gelten.

(ii) Die empirische Anforderung ist: Die W-Sätze sind an der günstigsten Stelle des Sprachverhaltens zu überprüfen, d.h. die propositionalen Einstellungen sind als ein Verhaltensdatum, somit als eine Beziehung von Sprechern und Sätzen direkt zu beobachten.

Die Belegbasis ist somit nicht die Reizbedeutung, da nur Überzeugungen weitere Gründe für andere Überzeugungen sein können. Deshalb stehen nicht basale Überzeugungen oder Beobachtungssätze, die sie ausdrücken, in einer epistemischen Beziehung zu einem neutralen Input. Eine solche Beziehung hält Davidson für unverständlich. Die Beobachtungssätze stehen nur in kausalen Beziehungen zu sensorischen Reizungen. Insofern können Reizungen nicht die Belegbasis für unsere Theorie über die Welt sein.

Es ist bereits an dieser Stelle hervorzuheben, dass "The theory describes conditions under which an utterance of a speaker is true, and so says nothing directly about what the speaker knows" [Davidson, 1990, S. 312]. Daran erkennen wir, dass sie die epistemischen Anforderungen der Interpretation gerade nicht spezifizieren kann.

(b) Die Interpretationstheorie hat den Zirkel zwischen Überzeugung und Bedeutung zu unterbrechen. Es ist somit eine einfache Einstellung herauszufinden, die ein Interpret auf der Seite des Sprechers annimmt, bevor er eine genaue Kenntnis der Einstellung des Sprechers hat. Die wahrheitsbedingte Bedeutungstheorie geht davon aus, dass die Einstellung einen „Satz für wahr-halten“ als Beleg (evidence), auf 
alle geäußerten Sätze anwendbar ist. „I propose that we take the fact that speakers of a language hold a sentence to be true (under observed circumstances) as prima-facie evidence that the sentence is true under those circumstances" [Davidson (1974) 1984, S. 152]. Das ist eine Meinung, deren Zuschreibung weder erfordert, dass der Interpret die Bedeutung des geäußerten Satzes kennt, noch dass er weiß, was der Inhalt der Meinung ist, die der Sprecher äußert. Einen Satz für-wahr-halten ist eine extensionale Beziehung zwischen einem Sprecher und einem Satz, aber kein intensionaler Zustand, der durch die Beziehung zu dem propositionalen Gehalt individuiert ist.

2. Die holistische Erfüllungstheorie der propositionalen Einstellungen erfordert einen Holismus derart, dass

(a) die meisten der propositionalen Einstellungen wahr sind und

(b) die rekursive Theorie der Bedeutung holistisch ist, d.h. sie ist auf jeden Satz einer Sprache zu spezifizieren. Insofern ist auch die Theorie der Überzeugungen holistisch. Der Holismus der wahren Überzeugungen soll als eine Adäquatheitsbedingung der Einstellungszuschreibung den globalen Skeptizismus (Descarte, Hume) widerlegen.

(c) Die Voraussetzung der wahrheitsbedingten Bedeutungstheorie der RI ist die unbegrenzte Anwendung des Grundsatzes der Nachsicht. Die RI schränkt den Grundsatz nicht auf eine Untermenge objektsprachlicher Sätze ein, z.B. auf Beobachtungssätze (Gelegenheitssätze) und ihre Wahrheitsfunktionen, sondern setzt ihn als einen allgemeinen Grundsatz ein, d.h. die Anwendung des Grundsatzes ist nicht nur ein erster Schritt in der Interpretation, sondern wir können keinen Sprecher erfolgreich interpretieren, wenn wir davon ausgehen, dass seine meisten Überzeugungen falsch sind.

3. Ist jedoch die Bedeutungstheorie als Wahrheitstheorie erfolgreich durchzuführen? Die Interpreten sind sich weitgehend darüber einig, dass Davidsons Ansatz gescheitert ist. Er führt werkgeschichtlich einige Revisionen durch, z.B. haben die Bikonditionale der W-Sätze nicht nur wahr zu sein, sondern ihnen ist der Status von Naturgesetzen zuzuweisen, um Konditionale wie z.B. ",,Schnee ist weiß" $g d w$ Gras grün ist" auszuschalten [Vgl. Davidson, (1976), 1984, S. 174, 171-179].

Seit der Kritik von John A. Forster (1976) und der Antwort von Davidson (1976) ist man sich über das grundlegende Problem dahingehend einig, dass die Theoreme der Form (B) $s$ bedeutet, dass $p$ (als ein 
intensionales Theorem) durch die extensionale Struktur des Satzes (W) $s$ ist wahr $g d w p$ nicht ersetzt werden kann [Forster 1976, S. 1-32; Davidson (1976) 1984, S. 171-179; Lepore, Ludwig, S. 113-118]. Das gilt auch dann, wenn wir wissen, dass der Satz, der , $p$ ' ersetzt, den Satz $s$ übersetzt. Insofern stellt sich zwangsläufig die Frage, ob man von der Wahrheit zur Bedeutung übergehen kann, da ein W-Satz nicht aussagt: $s$ bedeutet, dass $p$, d.h. er stellt nicht die propositionale Struktur der Sprache dar. Das wäre auch dann der Fall, wenn der Interpret weiß, dass die Axiome und Theoreme der Wahrheitstheorie als Gesetze einzustufen sind und die gebrauchten Sätze auf der rechten Seite des Konditionals dieselbe Bedeutung haben, wie die erwähnten Sätze auf der linken Seite des Konditionals.

Davidsons Fluchtpunkt ist deshalb der Schritt zu der Replacement Theorie, da er einräumt: „A theory of truth, no matter how well selected, is not a theory of meaning, while the statement that a translation theory entails certain facts is not, because of the irreducible indexical elements in the sentences that express it, a theory in a formal sense." Er schränkt das Zugeständnis dann im nächsten Satz ein: „This does not, however, make it impossible to say what it is that an interpreter knows, and thus to give a satisfactory answer to one of the central problems of the philosophy of language" [Davidson (1976) 1984, S. 179]. Wenn wir die epistemischen Anforderungen an die Aufgabe der „verständlichen Wiedergabe“ (intelligible redescription) der Sprechakte und der Propositionen eines Sprechers akzeptieren, so lässt sich die Frage nicht so stellen.

4. Lepore und Ludwig [2005, S. 19-37, 74-76] haben die Werkgeschichte Davidsons in systematischer Absicht rekonstruiert, um das grundsätzliche Problem seiner Wahrheitstheorie als Bedeutungstheorie zu identifizieren.

(a) Sie gehen auf die frühe Geschichte seiner Sprachtheorie ein, da man ohne sie den Fortgang seiner Theoriebildung insgesamt nicht angemessen rekonstruieren kann. Sofern man diesen Hintergrund nicht berücksichtigt, kann man ihnen zufolge nicht erklären, dass eine Wahrheitstheorie eine kompositionalen Bedeutungstheorie für primitive und komplexe Ausdrücke einer Sprache aufzustellen hat. Sie gehen davon aus, dass Davidson in "Theories of Meaning and Learnable Language" [1966] das auslösende Projekt (initial project) der Konstruktion der kompositionalen Bedeutungstheorie natürlicher 
Sprachen einführt hat und in „Truth and Meaning “ [1967] das umfangreichere Projekt (extended project) vorschlägt, dass mit einer extensionalen Wahrheitstheorie im Stile Tarskis eine Bedeutungstheorie aufstellbar ist [Davidson, (1966) 1984, S. 3-15, T (1967) 1984, S. 17-36]. Sie soll die übliche (deskriptive) Bedeutung der Terme einer Sprache erklären.

(b) Aus Lepores und Ludwigs Sicht ist die Durchführung des auslösenden Projekts davon unabhängig, was die Äußerungen eines Sprechers bedeuten. Damit wenden sie sich gegen die Replacement-Theory, die Davidson selbst nahelegt. ${ }^{25}$ Sie besagt, dass das auslösende durch das umfangreichere Projekt ersetzt wurde.

Das umfangreichere Projekt wird von ihnen im Unterschied dazu dahingehend beschrieben, dass es eine systematische Erklärung der Bedeutung von Sätzen beansprucht, in denen primitive Ausdrücke vorkommen. Lepore und Ludwig argumentieren dahingehend, dass die Replacement-Theory deshalb zurückgewiesen werden kann, sofern auf ihren Entstehungszusammenhang aus dem auslösenden Projekt zurückgegangen wird. Es betrifft dies die grundlegende Frage, ob eine Bedeutungstheorie als eine Wahrheitstheorie aufgestellt werden kann. Entscheidend ist bei dem Schritt zu dem umfangreicheren Projekt die Klärung des Bedeutungsbegriffs. Sie ist werkgeschichtlich für die Fortschreibung und die Veränderungen des gesamten Ansatzes in systematischer Absicht grundlegend. Das Problem des Ansatzes besteht (auch) darin, dass Davidson einen dispositionalen Bedeutungsbegriff hat, d.h. der Sprecher hat die Disposition, in bestimmten Situationen der Äußerung von bestimmten Sätzen, die kausal ausgelöst sind, zuzustimmen resp. sie zu äußern. Das Verfügen über Begriffe ist dadurch nicht zu erklären.

\footnotetext{
${ }^{25} \mathrm{Zu}$ der endgültigen Beziehung zwischen Kompositionalität als Methode und der Ersetzung durch die Wahrheitstheorie [Davidson, 1990, S. 299-301]. Er versucht die Einwände gegen die Bedeutungstheorie als Wahrheitstheorie dadurch zu entkräften, dass es ein Irrtum ist, die einmal aufgestellte Theorie im Hinblick auf die angemessene Reihenfolge der Erklärungsschritte mit der Erklärung der Richtigkeit der Theorie zu verwechseln. Das führt aber zu dem Problem, wie streng der Kompositionalitätsgrundsatz angewandt wird. Vgl. dazu [Lepore, Ludwig, 2005, S. 101-112].
} 


\section{Basistheorie der RI und Korrektur}

\section{(a) Interpretationsproblem}

Das Interpretationsproblem der RI besteht darin, dass der radikale Interpret festzustellen hat, ob ein W-Satz „s ist wahr $g d w$ p“ wahr ist, ohne zu wissen, was der erwähnte Satz $s$ im ersten Teil des Bikonditionals bedeutet. Das ist das grundlegende Problem des ganzen Ansatzes, das durch die Anwendung des Grundsatzes der Nachsicht gerade nicht zu lösen ist. Das wurde von vielen Davidsonkritikern herausgestellt. ${ }^{26}$

Damit ist die Basistheorie der RI angesprochen, da es die Interdependenz der beiden explanatorischen Faktoren Überzeugung und Bedeutung ist, die es verunmöglicht, ihre Rollen in der Interpretation sprachlichen Verhaltens voneinander zu unterscheiden. Der Umstand, dass ein Sprecher einen Satz für wahr hält, hängt davon ab, was der Satz in der betreffenden Sprache bedeutet, und zugleich davon, wovon der Sprecher überzeugt ist. Wenn wir davon ausgehen würden, dass der radikale Interpret nicht nur wüsste, welchen Satz der Sprecher für-wahr-hält, sondern auch, welche Bedeutung er hat, dann könnten wir seine Überzeugungen erschließen. Dazu verhält sich symmetrisch, wenn wir wüssten, welche Überzeugungen der für wahr gehaltene Satz ausdrückt, dann könnten wir erschließen, was er bedeutet. Die Schwierigkeit besteht gerade darin, dass die Daten, welche die RI stützen sollen, keine Informationen über Bedeutungen und Überzeugungen enthalten können. Die Folgerung daraus ist, dass die RI in einem Zug eine Theorie der Überzeugung und der Bedeutung aufzustellen hat.

Um zu plausibilisieren, dass wir Gründe dafür haben können anzunehmen, dass $s$ wahr ist, noch bevor wir seine Bedeutung kennen, geht der radikale Interpret, im Anschluss an Quine, von zwei Annahmen aus:

\footnotetext{
${ }^{26}$ Lepore und Ludwig heben z.B. hervor: „The principle of charity ... is introduced because patterns among hold true attitudes, as we have just noted, are not sufficient to fix one of the two factors which determine hold true attitudes (belief and meaning), to solve for the other. The principle of charity, in its role in fixing one of belief or meaning so that we can solve for the other, given hold true attitudes, requires support beyond any provided by abstract considerations governing coherent patterns of beliefs, meaning, and hold true (and other) attitudes. It will be, of course, crucial, then, to see what sort of justification can be provided for the principle introduced to do the job" [Lepore, Ludwig 2005, S. 185].
} 
1. Der radikale Interpret kann herausfinden, dass bei dem Sprecher z.B. ein assertorischer Sprachgebrauch vorliegt und zwar auch dann, wenn er über den kognitiven Inhalt der Aussage nicht informiert ist: „We can know that a speaker holds a sentence to be true without knowing what he means by it or what belief it expresses for him. But if we know he holds the sentence true and we know how to interpret it, then we can make a correct attribution of belief. Symmetrically, if we know what belief a sentence held true expresses, we know how to interpret it"[Davidson, (1975) 1984, S. 162] ${ }^{27}$.

2. Der radikale Interpret wendet den Grundsatz der Nachsicht an, d.h. er geht davon aus, dass die meisten Überzeugungen des Sprechers von seinem Standpunkt aus wahr sind. Damit ist die Annahme verbunden, dass die für-wahr-gehaltenen Sätze logisch konsistent sind.

Das grundsätzliche Problem der RI besteht darin, wenn der radikale Interpret nicht von der ersten Annahme ausgehen würde, so könnte er die Äußerung $A$ in der Sprache $x$ nicht identifizieren, über die sich das Wahrheitsprädikat erstreckt. Es bestünde dann nicht die Möglichkeit, seine Extension zu spezifizieren. Eine Semantik natürlicher Sprachen wäre dann aufzugeben. Das methodologische Problem der RI besteht darin, dass das, was von einem Interpreten direkt beobachtet wird, das Sprachverhalten in seiner Beziehung zu einer Umgebung, aus unbeobachtbaren Faktoren besteht: der Überzeugung und der Bedeutung.

Die zweite Annahme betrifft die epistemische Position des radikalen Interpreten. Damit geht der Anspruch einher, dass die Theorie der Interpretation beides, die Bedeutung der gesprochenen Wörter und den Inhalt der Einstellungen zu erklären hat. RI nimmt somit eine A priori-Struktur für die Analyse des Begriffs der Bedeutung, der Wahrheit, der Referenz sowie der Erfüllung an und gleichzeitig für die Überzeugungen, Absichten, Präferenzen, Handlungen als auch Evaluationen. Aus der Sicht der RI und ihrer Interpretationstheorie haben wir nur dann eine Chance die verschiedenen Äußerungen eines Sprechers zu interpretieren, wenn der radikale Interpret diese A priori-Rationalitätsannahme über den Sprecher vornimmt. Der Grundsatz der Nachsicht ist ein Brückengrundsatz der Anwendung der Wahrheits-Bedingungstheorie der Bedeutung. Sie soll möglich und erfolgreich sein, was

${ }^{27}$ Um diese Aufgabe zu lösen bedarf es aus dieser Sicht eine „verbundene Theorie“ (composite theory), die sprachliches und nichtsprachliches Handeln zu erklären vermag, [Davidson, (1975) 1984, S. 162-163]. 
auch immer über den Sprecher wahr ist. Die Systematisierung des Standpunkts des radikalen Interpreters und die distale Individuation der propositionalen Inhalte beansprucht, den Cartesianismus und dem Empirismus zu widerlegen.

Fassen wir die Basistheorie der RI zusammen, so ergibt sich folgendes Bild:

1. der Annahme eines rationalen Sprechers und Handelnden,

2. die mentalen und linguistischen Begriffe sind theoretische Begriffe,

3. die Einstellung der dritten Person der RI ist die begriffliche Basis für das Bedeutungsverstehen und die Zuschreibung von propositionalen Einstellungen,

4. das Verfügen über Gedanken ist sprachabhängig, insofern können nur Sprecher über ein Begriffsschema verfügen, d.h. wir identifizieren Begriffsschema mit übersetzbaren Sprachen und

5. es liegt eine vollständigen Information über die physische Interaktion zwischen dem Sprecher und dem Interpret und der beide umgebenden Umwelt vor.

6. Davidson, auch Quine und Richard Rorty, gehen davon aus, dass die Einstellung der dritten Person des Interpreten „global“ ist und die Grundlage der Interpretation und der Erkenntnistheorie. Davidson spricht von einer neuen Fassung der privilegierten Position des Interpreten. Das betrifft die epistemische Rolle des radikalen Interpreten, der seine eigenen Rationalitätsstandards und seine Reaktionen auf den Sprecher systematisch überträgt (Korrespondenzgrundsatz in der Triangulation) ${ }^{28}$

7. Die Überführung der RÜ in die RI beansprucht, dass soll aus dem holistischen Ansatz folgen, eine Begründung für die Unerforschbarkeit der Referenz zu geben und sie dadurch „erneut aufzustellen“. [Davidson, (1979) 1984 S. 241, 227-241]. Davidson nennt dies die Unbestimmtheit der Interpretation.

Zu der „Unbestimmtheit der Interpretation“ möchte ich noch etwas anmerken, da sie das zentrale Problem des Umbaus der Basistheorie von Quines RÜ betrifft. Angesprochen ist damit die Zurückweisung der ontologischen Relativität. Davidson spricht statt dessen von der „Relativität der Bezugnahme“ [Davidson, (1979) 1984 S. 228]. Da Davidson die Stimulusbedeutung und die ontologische Relativität ablehnt, reinterpretiert er die Unbestimmtheit der Übersetzung derart, dass sie auf der Unerforschbarkeit der

\footnotetext{
${ }^{28}$ Siehe dazu die kurze Zusammenfassung [Davidson, 2001, S. 211-213]
} 
Referenz, der Unbestimmtheit von unten, „unmittelbar ... folgt“ [Davidson, (1979) 1984 S. 228]. Das ist unbezweifelbar keine richtige Interpretation von Quines Ansatz. Die Folgerung ist von dem Standpunkt der RI und ihren Voraussetzungen aus folgerichtig. Sie besagt, dass die mentalen Begriffe theoretische Begriff sind, die durch die Interpretationstheorie definiert sind und viele Theorien sollen diese Aufgabe erfüllen können (Kritik an der kausalen Referenztheorie der Namen und allen sogenannten „Bausteintheorien“). Der Theorierelativismus soll dabei ausgeschaltet werden. Das ist dadurch begründet, dass die Unbestimmtheit der Übersetzung von oben relativiert ist, da eine erfolgreiche radikale Interpretation als Ergebnis einen adäquaten Begriff der Synonymie von Äußerungen bereitstellt. Die Äußerung der Sätze „Schnee ist weiß" von unterschiedlichen Sprechern besagen dann dasselbe. Sie sind "Gleichsager“ (samesayer). Es betrifft das die „Inhaltssätze“, die auf „sagen, dass" folgen. Nach Davidson verschiebt sich das Problem der Unbestimmtheit der Übersetzung zu Gunsten der Unterbestimmtheit der (Wahrheits-) Interpretationstheorie und ihrer Axiome. Das grundsätzliche Problem dieser Reinterpretation ist es, dass aus der epistemische Position des Interpreten bei der Anwendung von Begriffen (Klassifikationen) auf irgendetwas nicht gefolgert werden kann, dass sie theoretisch sind, d.h. durch die (Wahrheits-) Interpretationstheorie implizit zu definieren sind.

\section{(b) Einwände}

Wenn das Für-wahr-halten der Beleg für die Zuschreibung eines propositionalen Inhalts sein soll, dann kann der Beleg kein Verhaltensdatum im Sinne eines offensichtlichen Verhaltens (overt behavior) sein, d.h. die Wahrheitswerte und das Für-wahr-halten sind keine untheoretische Beschreibung eines beobachtbaren Verhaltens. Es ist dagegen einzuwenden:

1. Für Davidsons Interpretationstheorie gilt, vergleichbar mit Quines RÜ, dass sie die Korrelationen zwischen dem Für-wahr-halten und den Umweltbedingungen nicht eindeutig qualifizieren kann, da Mengen von Korrelationen und inkompatible Interpretationstheorien vorliegen können.

2. Das Argument des Grundsatzes der Nachsicht als eine A priori-Annahme begründet die Einstellung der dritten Person nicht, sondern setzt sie voraus. Es ist zu erwähnen, dass die Quine-Davidson Tradition H.-N. Castañedas (P. Geach) Entdeckung der „quasi indication“ systematisch 
nicht verarbeitet hat. Das ist der blinde Fleck ihrer Umweltbeobachtung [Castañeda, '(1966), 1999, S. 35-60].

3. Es ist zwischen der Verfügung über den Begriff der objektiven Wahrheit und der Identifizierung eines eindeutigen Gegenstandes der Sprechergedanken zu unterscheiden.

Es bleibt unbeantwortet „Was sind die Gegenstände von Gedanken (propositonale Gehalte, Frege-Gedanken)?"،. Die Frage wird aus meiner Sicht absurd beantwortet, da die Gegenstände der Gedanken die raum-zeitlichen Gegenstände selbst individuiert sein sollen. Es ist aber kaum zu bezweifeln dass die de re Überzeugungen kein de se und keine de dicto Zuschreibungen implizieren. Eine Cartesianische Auffassung, ohne einen ontologischen Dualismus, ist derart zu verteidigen, dass der subjektive Standpunkt impliziert, dass wir einen unmittelbaren Zugang zu unseren eigenen Gedanken und dem Mentalen haben, der uns phänomenal bewusst ist. Es ist insofern auch nicht einzusehen, dass unsere semantische Intuition für jede Interpretation einfach hinfällig und als Museumsmythos zu entzaubern ist. Diese Intuition ist durch das öffentliche Merkmal als das semantische Merkmal der Sprache gerade nicht außer Kraft gesetzt. Insofern ist die Beziehung zwischen „Bedeutung“ und "Öffentlichkeit" neu zu fassen.

4. Es liegt ein fast trivialer Einwand gegen die unbegrenzte Anwendung des A priori-Grundsatzes der Nachsicht nahe. Das gilt sowohl für seine Anwendung im Fall der RÜ als auch der RI. Sollte die Situation eintreten, dass sich der Sprecher fortlaufend irrt, so tritt das Interpretationsproblem auf, dass der Sprecher, z.B. bei einer uneinheitlichen Verhaltensweise, d.h. er stimmt unsere Übersetzung einmal zu und in anderen Fällen lehnt er sie ab, von dem Übersetzer/Interpreten ein Irrtum zugeschrieben wird.

Das gilt unter der Voraussetzung, dass die Übereinstimmung zwischen Sprecher und Interpret durch die Interpretationstheorie im Hinblick auf die Umstände der Äußerungssituation festgelegt ist und wir die weitgehende Widerspruchsfreiheit seiner Einstellungen anzunehmen haben. Die Einräumung eines Irrtums bei Beibehaltung der homophoner Übersetzung bzw. der Beibehaltung der Interpretationstheorie beansprucht somit eine Verhaltenserklärung, die von den offensichtlichen Umständen unterschieden ist. Das ist an dem Beispiel der Hasen als Boten von fremden Göttern gut zu erkennen. Davidson versucht das Problem dadurch zu lösen, dass viele Interpretationstheorien ihre Auf- 
gabe erfüllen können. Gehen wir davon aus, dann lässt sich die Übereinstimmung und Nichtübereinstimmung dieser Theorien zwischen Sprecher und Interpret nicht mehr auf der Basis der Verhaltensbelege gewährleisten. ${ }^{29}$

Es ist hervorzuheben, dass, um überhaupt den Grundsatz der Nachsicht anwenden zu können, zwischen:

(a) dem relevanten Wissen der Sprecher und Interpreten als auch ihrem Hintergrund sowie der Art ihres Zugangs zu ihm,

(b) der Zuschreibung von bestimmten sprachlichen und nicht-sprachlichen Handlungen und

(c) der Zuschreibung von unterschiedlichen Einstellungen im Hinblick auf die Äußerungssituation und ihre Definition zu unterscheiden ist. Die Definition der Situation betrifft den sozialen Rahmen der Einstellungszuschreibung, der gerade nicht zu naturalisieren ist. Daraus folgt aber eine weitgehende Umschichtung des Grundsatzes der Nachsicht zu dem Grundsatz der epistemischen Gerechtigkeit, d.h. wir unterstellen, dass der Sprecher von seinem Standpunkt aus Recht hat, solange nicht das Gegenteil vorliegt [Preyer, 2011a, S. 33, 62, 109, 113-114].

4. Insofern kann das Bezugsproblem der Übersetzung, der Interpretation als auch der reinterpretativen Strategie der Wahrheit und anderer Erfüllungsbedingungen von Sätzen und illokutiven Akten gerade nicht eine einfache Korrespondenz mit physischen Tatsachen (Nettobedeutung) oder der distale Inhalt der Einstellungen eines Sprechers sein.

$\mathrm{Zu}$ erwähnen ist auch, dass der Grundsatz der Nachsicht bei seiner Verfeinerung zunehmend im Hinblick auf die epistemische Qualifikation des Sprechers feinkörnig wirkt, z.B. was weiß der Sprecher, was nimmt er an, was hält er für möglich und soll er sich aufrichtig verhalten. Das ist in der mir bekannten Literatur nicht vermerkt worden. ${ }^{30}$ Es sind zusätzliche Annahmen als zusätzliche Anforderungen für die verständliche Neubeschreibung

${ }^{29}$ Davidson hat keine über die Wahrheitstheorie hinausgehende reinterpretative Strategie der „intelligible Redescription“.

${ }^{30}$ In den Diskussionen mit M. Roth sind wir darauf gestoßen. Davidson [1990, S. 298-299] lehnt, von seinem Ansatz folgerichtig, eine epistemische Bestimmung grundsätzlich ab, sie ist aus seiner Sicht ein „Irrtum“. Sie lädt zum Skeptizismus ein und zwar nicht, da sie behauptet, dass die Realität unerkennbar sei, sondern weil sie Realität auf unsere Überzeugungen über 
des Sprechers und Handelnden heranzuziehen, z.B. seine Psychologie, seine Kultur und sein sozialer Status. Die Interpretation ist nicht einfach dadurch gewährleistet, dass er ein Sprecher ist. Der epistemische Gesichtspunkt der RI als eine Rechtfertigung der interpretativen Wahrheitstheorie für Sprecher auf der Basis der Anwendung des Grundsatzes der Nachsicht ist somit zu dürftig, um dem zu interpretierenden Sprecher ein qualitativen Wissen zuzuschreiben und die distale Bedeutungstheorie kann die Kluft zwischen den Überzeugungen des Interpreten und dem Sprecher nicht schließen. Insofern ist Lepore und Ludwig zustimmen, dass das ,anspruchsvolle Projekt“ der RI, d.h. jeder Sprecher ist von Natur aus radikal interpretierbar, nicht einzulösen ist. Einlösbar ist nur das „bescheidene Projekt“, d.h. es ist eine empirische Frage im Hinblick auf den Sprecher, ob er radikal interpretierbar ist oder nicht. Aus dieser Sicht ist die Davidson-RI letztlich unmöglich [Lepore, Ludwig 2005, S. 151-173; Lepore, J. A. Fodor, 1993, S. 57-76]. Der Umstand, dass einige Sprecher radikal interpretierbar sind, ist seinerseits eine epistemische Anforderung, da keine Interpretation die „epistemische Kapazität“ der daran beteiligten übersteigen kann.

\section{(c) Konvention W}

Die interpretative Wahrheitstheorie teilt mit Quine den Verzicht auf Propositionen (Intensionen). Für Quine und Davidsion gilt, dass es für die Bedeutungstheorie keine Eigenschaften gibt, wie z.B. Frege-Sinne, Propositionen oder semantische Universalien. Davidson argumentiert dahingehend, dass man zwar Propositionen akzeptieren mag, das ist eine Sache der Entscheidung, aber sie sind ohne Nutzen. ${ }^{31}$ Es ist ihr Anspruch den Irrweg der Zulassung von Propositionen (David Amstrong: Wahrheitsträgern) in der Sprachtheorie und Semantik zu korrigieren. Angesprochen ist damit auch ein zentrales Problem von Tarskis Wahrheitsbegriff, der Wahrheit auf eine bestimmte Sprache spezifiziert. Demnach sind Sätze wahr oder falsch und nichts anderes. Wenn diese Position nicht haltbar ist, dann ist auch die interpretative Wahrheitstheorie nicht überzeugend. Das gilt dann, wenn wir

sie zurückführt. Das ist für eine epistemische Qualifikation nicht zwingend, da sie keinen erkenntnistheoretischen Idealismus annimmt.

${ }^{31}$ Dazu [Lepore, Ludwig, 2005] Capt. 3. Über Propositionen als die Bedeutung von Sätzen in Sätzen mit „That clauses“ (Noun phrase + verb + that + sentence) [Lepore, Ludwig, 2005] Donald Davidson Truth-Theoretic Semantics, capt. 11. 
Propositionen als Wahrheitsträger in der Semantik zulassen. Es ist deshalb danach zu fragen „Warum benötigen wir Propositionen?“.

Es ist empfehlenswert im Hinblick auf die Zulassung von Propositionen Arthur Paps Ansatz in Erinnerung zu rufen. Was ist die Relation zwischen dem semantischen Inhalt '"p' ist wahr" und der Aussage über eine Proposition „W(p)“? [Pap, 2006, S. 67, (1953), S. 148-154, (1954), S. 155-164, 165-179]. Er vergleicht „W(p)“ mit der Beziehung zwischen der Aussage "Das Prädikat , $P$ ' ist auf $x$ anzuwenden“. Es mag sein, dass $x$ die Eigenschaft $P$

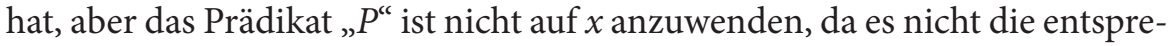
chende Eigenschaft hat. Pap schließt daraus, dass die empirische Wahrheit die Exemplifikation einer Proposition durch bestimmte Tatsachen ist, so wie die Exemplifizierung von Universalien oder Attributen durch Individuen. Wenn irgendeine Proposition $p$ wahr ist, dann sind aber noch nicht die Tatsachen festgestellt, welche die Proposition wahr macht. Wenn die Proposition, dass es rote Autos auf der Erde gibt wahr ist, so ist noch nicht etwas darüber gesagt, wie viele Autos rot sind und wo sie sich befinden [Bayleis, 1948, S. 459-479]. Der Begriff der Wahrheit ist in unserem Alltagsverständnis ein nichtsemantischer Begriff, so wie die Exemplifizierung von Eigenschaften und der Teile von Klassen. Die Sätze, wie z.B. „Schnee ist weiß ist wahr“ oder "Schnee ist weiß ist falsch" haben einen Inhalt. Insofern gibt es etwas, das die Sätze wahr macht (David Armstrong: truth-maker). Diese Sätze selbst sind als Sätze leer. ${ }^{32}$ Dazu ein Hinweis.

Das Problem einer Wahrheitstheorie im Stile Tarski's ist, dass die logische Äquivalenzen der Form Wahrheit, wie z.B. „Porto ist eine Stadt in Portugal ist wahr $g d w$ Porto eine Stadt in Porto ist" keine notwendig wahren Sätze sind. Wir können uns ohne einen Widerspruch zu begehen vorstellen, auch dann, wenn Porto eine Stadt in Portugal ist, dass der Satz „Porto ist eine Stadt in Portugal“ nicht wahr ist. Der Satz würde dann keine wahre, sondern eine falsche Proposition bezeichnen. Dies könnte z.B. der Fall sein, wenn ein Sprachwandel in einer sozialen Gruppe eintritt und dadurch der Satz eine andere Proposition bezeichnet. Von der Proposition Porto ist eine Stadt in Portugal sind andere Propositionen zu folgern, z.B. Porto ist das Wirtschaftszentrum von Portugal. Das gilt dann, wenn der Satz „Porto ist das Wirtschaftszentrum von Portugal" ein analytischer Satz ist oder im Falle der Existenzunterstellung „Es gibt nur ein einziges Wirtschaftszentrum in Portugal“. Es ist aber

\footnotetext{
${ }^{32}$ In diese Richtung argumentiert auch J. R. Searle.
} 
nicht zu folgern, dass der Satz „Porto ist eine Stadt in Portugal“ die Proposition Porto ist eine Stadt in Portugal bezeichnet.

Insofern ist der Wahrheitswert der Proposition „Porto ist eine Stadt in Portugal“ davon abhängig, welche Proposition durch den Satz bezeichnet wird, während der Wahrheitswert der Aussage über Porto nicht von den semantischen Tatsachen abhängt. Von dem Satz „wahr $(, p, L)^{\prime \prime}-\mathrm{L}=$ Sprache - ist zu folgern „, $\exists x)$ wahr $(x, L)$ “: Es gibt Sätze, insbesondere den Satz $p$, aber aus $p$ ist nicht irgendein Satz zu folgern. Wir können uns eine Situation vorstellen, in der die Äquivalenz „„,Porto ist eine Stadt in Portugal“ ist wahr $g d w$ Porto ist eine Stadt in Portugal“ ein falscher Satz ist. Daher sind die Äquivalenzen der Form Wahrheit im Stile Taskis keine Adäquatheitsbedingung der Wahrheitsdefinition [Pap, 2006, S. 64-65, 68].

Für die Definition der Wahrheit benötigen wir das Wort „Tatsache“, mit dem wir aussagen, dass eine Proposition wahr ist. Ein wahre Aussage entspricht den Tatsachen. Tatsachen sind keine Objekte, aber durch den Gebrauch des Wortes sagen wir erfüllte Wahrheitsbedingungen aus. Die erfüllte Wahrheitsbedingung einer Proposition ist eine Tatsache. Dadurch entspricht die erfüllte Wahrheitsbedingung einer Tatsache, welche eine wahre Proposition spezifiziert. Die Tatsache, dass Porto eine Stadt in Portugal ist, erfüllt somit die Wahrheitsbedingung einer nicht-sprachlichen Proposition. Es ist somit nicht die Eigenschaft eines Satzes, welche eine Proposition wahr oder falsch macht: Eine Aussage ist wahr oder erfüllt $g d w$ sie mit den Tatsachen entspricht.

\section{Blick auf einen alternativen Ansatz}

Die vorgenommene Darlegung im Hinblick auf den Bezugsrahmen von RÜ und RI ist überwiegend kritisch ausgefallen. Eine Würdigung der beiden Ansätze wurde erst einmal zur Seite gelegt. Ich möchte aber, um nicht beim Kritischen zu verbleiben, einen Hinweis auf einen alternativen Bezugsrahmen geben, in den wir das Mentale und das Sprachverhalten einordnen. In der Konsequenz führt dieser Schritt dazu, dass wir uns von der Kritik an dem „Dritten Dogma des Empirismus“ (Davidson), der Zurückweisung der Schema-Inhalt-Unterscheidung, distanzieren sollten ${ }^{33}$.

${ }^{33}$ Eine Kritik an der Kritik am „Dritten Dogma“ kann auch dadurch erfolgen, dass mit dem Ausdrucksreichtum unsere Sprache „reichhaltigere“ Inhalte gegeben sind. Das begründet aber gerade keinen linguistischen Relativismus. 
Die Analyse des Bezugsrahmens der Interpretation sollte von der Annahme von primitiven Fähigkeiten aus erfolgen. In dem Bezugsrahmen dieser Fähigkeiten verorten wir beide Positionen, die des Sprechers und des Interpreten. Dabei empfiehlt es sich den allgemeinen Bezugsrahmen von seiner Lokalisierung zu unterscheiden. Insofern lässt sich die Unterscheidung zwischen einem Tiefen- und einem lokalen Hintergrund treffen. Die Analyse des angesprochenen Bezugsrahmen setzt die Innen-Außendifferenzierung zwischen des Standpunkt des Subjekts (Denkenden, Sprechers, Handelnden) und der Außenwelt voraus. ${ }^{34}$

1. Wir unterstellen einen Begriff der inneren Welt, d.h. wir haben Bewusstsein und ein Zeitbewusstsein. Das ist eine Voraussetzung dafür, dass wir Pläne entwerfen und Absichten ausbilden können. Die grundlegende Frage ist dabei „Was ist das charakteristische Merkmal des Bewusstseins?". Das Problem ist, eine nicht zirkuläre und regressfreie Antwort auf das Bewusstseinsmerkmal zu geben. Verbreitet ist die Argumentation, vergleichbar im Hinblick auf das Zeitbewusstsein, dass eine zirkelfreie Definition nicht gegeben werden kann.

2. Dieses Merkmal besteht darin, dass wir über ein unmittelbares (präreflektives/irreflexives) Bewusstsein verfügen, durch das unsere innere Welt uns selbst bewusst ist. ${ }^{35}$ Die weitgehende Behauptung ist, dass wir nur deshalb über unsere Einstellungen nachdenken und zukünftige Ereignisse vorwegnehmen können. Präreflektives Bewusstsein bindet meine mentalen Zustände als meine Zustände. Es ist aber kein Ichbewusstsein. Dieses Bewusstsein macht uns erst zu einem Sprecher (Denkenden, Handelnden), der sich seiner mentalen Zustände bewusst ist.

3. Wir verfügen über eine mentale Sprache, in der wir unsere mentalen Zustände ausdrücken können. Sie ist privat und gerade nicht öffentlich (N. Chomsky, J. R. Searle: interne versus externe Sprache).

4. Wir verfügen über einen Begriff der Emotion, um z.B. Trauer und Freude zu verstehen sowie zur Empathie in der Lage zu sein. Die Emotionen

\footnotetext{
${ }^{34}$ [Gärdenfors, 2003, S. 83-109] geht in eine ähnliche Richtung. Die von mir vorgenommenen Unterscheidungen gehen von einem anderen Begriff des Selbstbewusstseins aus.

${ }^{35}$ Die Relevanz der Präreflektivität für die Verfasstheit mentaler Zustände hat vor allem M. Frank herausgestellt, z.B. [Frank, 2012; 2015]. Dazu [Preyer, 2013a, 2015]. Academia Gerhard Preyer, Einheit: Consciousness, Mind, [Miguens, Preyer, Morado, Bravo, 2015].
} 
sind dynamische Körperdispositionen, die uns immer total erfassen. Es ist subjektiv schwer, sie von ihren Wirkungen zu unterscheiden. ${ }^{36}$

5. Wir verfügen über den Begriff der Aufmerksamkeit, um etwas in und außerhalb von uns zu erfassen. Das ist eine Voraussetzung dafür, dass wir über eine innere Welt und die Unterscheidung zwischen Innen und Außen verfügen. Die Aufmerksamkeit auf etwas ist ihrerseits präreflexiv bewusst. $^{37}$

6. Wir verfügen über den Begriff der Intentionalität, der Absicht und des praktischen Denkens, um darüber zu entscheiden, in welchem Ausmaß dieses Denken erfolgreich sein kann oder nicht.

7. Die Unterscheidungen sind ihrerseits auf die Sprachfunktionen der Darstellung von Sachverhalten (assertive illokutive Typen), der Mitteilung (regulative illokutive Typen) und des Ausdrucks mentalen Zuständen (intentionaler Ausdruck) zu respezifizieren, in denen sie sich realisieren, aber nicht erschöpfen. Ihre Analyse setzt die Erfüllungsbedingungen von „offenen“ und "geschlossenen“ Propositionen (Dieter Wunderlich) voraus.

8. Wenn wir von primitiven Fähigkeiten und ihrem sprachlichen Ausdruck ausgehen, so setzt das einen Beobachter (Interpreten) voraus. D.h. die Unterscheidungen kommen sozusagen nicht von Außen auf uns zu, sondern sie sind Voraussetzungen, die wir ihrerseits nicht theoretisch aus Axiomen ableiten können. Die „reflexive Schleife“ auf einen Beobachter und die Beobachtung von Beobachtern (Niklas Luhmann) ist aber ihrerseits zu unterbrechen, da sonst ein Zirkel und ein Regress nicht $\mathrm{zu}$ vermeiden ist. ${ }^{38}$ Es lässt sich erkenntnistheoretisch nicht ausschließen, dass wir bei einer Paradoxie des Unterscheidens enden. Das

${ }^{36}$ Vgl. dazu auch die Untersuchungen z.B. von A. Damasio, J. LeDux, D. Goleman und P. Goldie. Goldie unterscheidet zwischen „body feelings“, z.B. schwitzen und Herzrasen und „feelings toward“, z.B. Freude, Wut und Neid. Letztere stuft er als intentional ein. Zu einer weitergehenden Analyse [Merker, 2012, S. 253-268]. Für die Emotionstheorie wäre es förderlich, wenn sie sich J.-P. Sartres, [(1936), 1964, S. 153-195]. Emotionsanalyse in Erinnerung rufen würde. Sie thematisiert die „Magie“ als emotive Objektbeziehung. Eine Emotion verweist auf das, was sie bedeutet. Sie setzt aber nach Sartre die „vorgängige Beschreibung der Affektivität voraus, insofern letzter das Sein des Daseins konstituiert, d.h. insofern es für unser Dasein konstitutiv ist, affektives Dasein zu sein“, S. 194, Zum Magischen [Sartre (1936), 1964, S. 189-190].

37 Das wurde als ein Grenzproblem der Phänomenologie erörtert. Dazu [Beils, 1987].

${ }^{38}$ Die zirkuläre Konstruktion von Kommunikation und sozialen Systemen ist insofern nicht die letzte Antwort. D. h. nicht, dass Ursprungsfragen gestellt werden. 
sollte uns aber nicht dazu verleiden, die externe Sprache und die erkenntnistheoretische Reflexion als unhintergehbar zu halten. Was das Letztere betrifft, so hat uns Manfred Frank die romantische Einsicht in Erinnerung gerufen: "Was die Reflexion findet, scheint schon da zu seyn" (Novalis).

Die in den Blick genommenen Unterscheidungen betreffen strukturelle Vergleichbarkeiten zwischen Sprecher und Interpret, die reinterpretative Strategien von sprachlichem und nichtsprachlichem Verhalten voraussetzten. Auf der Grundlage von dispositonalen Verhaltenskorrelationen und kausalen Beziehungen bleibt Fremdpsychisches und Sprachbedeutung unverständlich. Fodor hat die Hauptströmung der anglophonen Philosophie der Sprache, der Erkenntnistheorie und der Philosophie des Geistes seit der Mitte des letzten Jahrhunderts durch: 1. eine simple - (Quine) und 2. eine sophistische Verhaltenswissenschaft (Davidson) charakterisiert. Beide Traditionen, die von einer pragmatischen Version des Verfügens über einen Begriff (concept possession) ausgehen, sind nicht mehr zu innovieren. ${ }^{39}$

Beitrag zu Trans-Late - Über-Setzen - Tra-Duire. Language Diversity and Intercultural Hermeneutics Sprachdiversität und interkulturelle Hermeneutik. International Conference - Internationale Tagung - Congrès international, 15.-17. 5. 2015

\section{Literaturverzeichnis:}

Bayleis C. A. 1948. "Facts, Propositions, Exemplification and Truth", Mind 57: 59479.

Beils K. B. 1987. Transzendenz und Zeitbewusstsein. Zur Grenzproblematik des transzendental-phänologischen Idealismus. Bonn: Bouvier Verlag Herbert Grundmann.

Burge Tylor (1979) 2007. Individualism and the Mental. In. Foundation of Mind, 100-150. Oxford: Clarendon Press.

Burge Tylor. (1993) 2007. Mind-Body Causation and Explanatory Practice. In. Foundation of Mind, Oxford: Clarendon Press.

Burge Tylor. 2010. Origins of Objectivity. Oxford: Clarendon Press

39 [Fodor, 2004, S. 29-47]. In der Sprachtheorie/philosophie befinden wir uns mittlerweile in einer veränderten Problemsituation. Lepore, Fodor haben durch den semantischen, kompositionalen Atomismus einen "Neuen Linguistic Turn" vollzogen, [Fodor, 2002; Cappelen, Lepore, 2005; Preyer, Peter, 2007, 2005]. 
Cappelen, H., Lepore. 2005. Insensitive Semantics. A Defense of Semantic Minimalism and Speech Act Pluralism, Malden: Blackwell.

Castañeda, Hector Neri. (1966) 1999. 'He': A Study in the Logic of Self-Consciousness. In. The Phenomenology of the I. Essays on Self-Consciousness. Hart J. G., Kapitan T. (Hrsg.), 35-60. Bloomington, Indianapolis: Indiana University Press: Chomsky Noam. 1957. Verbal Behaviour By B. F. Skinner, Language 35 (1) 1959: 2658 Kritik an B. F. Skinner, Verbal Behaviour, Englewood Cliffs, NJ: Prentice-Hall. Chomsky Noam. (1969) 1975. Quine's Empirical Assumptions, in Davidson and J. Hintikka eds., Words and Objections. Essays on the Work of W. V. Quine. Dordrecht revised edition.

Davidson Donald. (1974) 1984. Belief and the Basis of Meaning. In. Davidson Donald. Truth and Interpretation. 141-154. Oxford: Clarendon Press.

Davidson Donald. (1975) 1984. Thought and Talk. In. Davidson Donald. Truth and Interpretation. Oxford: Clarendon Press.

Davidson Donald. (1976) 1984. Reply to Forster, 171-179. In. Davidson Donald. Truth and Interpretation. Oxford: Clarendon Press.

Davidson Donald. (1979) 1984. The Inscrutability of Reference. 240-241. In. Davidson Donald. Truth and Interpretation. Oxford: Clarendon Press.

Davidson Donald. (1980) 2004. A Unified Theory of Thought, Meaning, and Action. S. 151-166, In. Davidson Donald. Problems of Rationality. Oxford: Clarendon Press.

Davidson Donald. 1980. Essays on Actions and Events. Oxford: Clarendon Press.

Davidson Donald. (1982) 2004. Paradoxes of Irrationality. 169-187. In. Davidson Donald. Problems of Rationality. Oxford: Clarendon Press.

Davidson Donald. (1985) 2004. Incoherence and Irrationality. 189-198. In. Davidson Donald. Problems of Rationality. Oxford: Clarendon Press.

Davidson Donald. 1990. „The Structure and Content of Truth”. The Journal of Philosophy, Vol. LXXXVII, (6): 279-328.

Davidson Donald. 1984. Truth and Interpretation. Oxford: Clarendon Press.

Davidson Donald. 1999. Is Truth a Goal of Inquiry? Discussion with Rorty, S.18-19, In: U. M. Zeglen (Hrsg.) Donald Davidson. Truth, Meaning and Knowledge, London/New York: Routledge Publisher.

Davidson Donald. 1999. The Centrality of Truth. In J. Peregrin (Hrsg.) Truth and ist Nature (is any). 104-115. Dordrecht: Kluwer Academic Publishers.

Davidson Donald. 2001. Three Varieties of Knowledge. 211-213, In.. Subjective, Intersubjective. Objective, Oxford: Clarendon Press.

Davidson Donald. 2001. Subjective, Intersubjective. Objective, Oxford: Clarendon Press.

Davidson Donald. 2004. Problems of Rationality. Oxford: Clarendon Press.

Davidson Donald. 2005. Truth, Language, and History. Oxford: Clarendon Press. 
Davidson Donald. 2005. Method and Metaphysics. In: Davidson Donald. Truth, Language, and History. Oxford: Clarendon Press.

Fodor Jerry A. 2002. The Compositional Papers, Oxford: Clarandam Press.

Fodor Jerry A. 2004. "Having Concepts: a Brief Refutation of Twentieth Century". Mind \& Language, Vol. 19 (1) February: 29-47.

Forster J. A. 1976. Meaning and Truth, 1-32. In. Evans, G., J. McDowell (Hrsg.). Truth and Meaning: Essays in Semantics, Oxford: Clarendon Press.

Frank Manfred. 2012. Ansichten der Subjektivität. Berlin: Suhrkamp Verlag.

Frank Manfred. 2015. Präreflexives Selbstbewusstsein. Vier Vorlesungen, Stuttgard: Reclam.

Gärdenfors Peter. 2003. How Homo became Sapiens. On the Evolution of Thinking, Oxford: Oxford University Press.

Gerhard Preyer, Miguens Sofia (Hrsg.). 2013. Consciousness and Subjectivity, Berlin: De Gruyter.

Gerhard Preyer, Miguens Sofia, Clara Bravo Morando (Hrsg.). 2015. Pre-reflective Consciousness. Sartre and Contemporary Philosophy of Mind, London: Routledge.

Katz Jerrold J. (1966) 1969. Philosophie der Sprache. Frankfurt a. M. (Berlin): Suhrkamp Verlag.

Ludwig Kirk. 1999. Theories of Meaning, Truth and Interpretation. In. U. Zeglen (Hrsg.) Donald Davidson Truth, Meaning and Knowledge. London/New York: Routledge Publisher.

Lepore Ernie, Jerry A. Fodor. 1993. Is Radical Interpretation Possible? In R. Stoecker, Reflecting Davidson. Donald Davidson Responding to an International Forum of Philosophers, Grundlagen der Kommunikation und Kognition, Hrsg. R. Posner, G. Meggle, 57-76, Hawthorne: De Gruyter.

Lepore Ernie, Kirk Ludwig. 2005. Forster's Objection, 113-118. In. Lepore Ernie, Kirk Ludwig Donald Davidson. Meaning, Truth, Language, and Reality. Oxford: Clarendon Press.

Lepore Ernie, Kirk Ludwig. 2005. Donald Davidson Meaning, Truth, Language, and Reality. Oxford: Clarendon Press.

Lepore Ernie, Kirk Ludwig. 2007. Donald Davidson's Truth-Theoretic Semantics, Oxford: Clarendon Press.

Ludwig Kirk (Hrsg.). 2003. Donald Davidson, Contemporary Philosophy in Focus, Cambridge: Cambridge University Press.

MacDonald Graham, Pettit Philip. 1981. Semantics and Social Science, London: Routledge and Keagan Paul.

Merker Barbara. 2012. Kritik der Gefühle. In G. Peter, R.-M. Krausse, Selbstbeobachtung der modernen Gesellschaft und die neuen Grenzen des Sozialen, 253-268. Wiesbaden: Springer/VS Verlag. 
Pap Arthur. (1953). 2006. Analytische Erkenntnistheorie. Notes on the "Semantics" and the "Absolute" Concept of Truth, The Limits of Logical Empiricism. A. Keupink, S. Shieh (Hrsg.). Wien.

Pap Arthur. (1954) 2006. Proposition, Sentences, and the Semantic Definition of Truth. In The Limits of Logical Empiricism. A. Keupink, S. Shieh (Hrsg.). Wien.

Preyer Gerhard, M. Ulkan, A. Ulfig (Hrsg.). (1996). Intention, Bedeutung, Kommunikation. Kognitive und handlungstheoretische Grundlagen der Sprachtheorie

Preyer Gerhard et al. (Hrsg.) (1994) 2004. Language, Mind and Epistemology.

On Donald Davidson's Philosophy, Synthese Library (241), Wien: Springer.

Preyer Gerhard, Georg Peter. 2005. Contextualism in Philosophy. Knowledge, Meaning and Truth, Oxford: Clarandon Press.

Preyer Gerhard, Georg Peter, 2007 Context-Sensitivity and Semantic Minimalism. New Essays on Semantics and Pragmatics, Oxford: Oxford University Press.

Preyer Gerhard. 2011a. Donald Davidson's Philosophy. From Radical Interpretation to Radical Contextualism, Frankfurt a. M.: Humanities Online (zweite Auflage).

Preyer Gerhard. 2011b. Intention and Practical Thought, Frankfurt a. M.: Humanities Online.

Preyer Gerhard (Hrsg.). 2012a Donald Davison on Truth, Meaning, and the Mental, Oxford: Oxford University Press.

Preyer Gerhard. 2012b. Interpretation, Sprache und das Soziale. Philosophische Aufsätze, Frankfurt a. M.: Humanities Online (zweite Auflage).

Preyer Gerhard. 2013a. "Besprechung: Ansichten der Subjektivität", Zeitschrift für philosophische Forschung (1) .

Preyer Gerhard. 2013b. The Externalism of Triangulation. In. Amoretti, M. C. Preyer G. Triangulation. From an Epistemological Point of View, 197- 220. Berlin: De Gruyter.

Preyer Gerhard. 2014. Back to Cartesian Intuition. Internalism, Externalism and the Mental, Academia under Preyer unit consciousness, mind.

Preyer Gerhard. 2015. Subjektivität als präreflexives Bewusstsein. Jean-Paul Sartres „bleibende Einsicht“Zu Manfred Frank, Präreflexives Selbstbewusstsein. Vier Vorlesungen, Stuttgart: Reclam 2015, Academia under Preyer unit consciousness, mind.

Putnam Hilary. 1975. Mind, Language and Reality. Philosophical Papers (2 Vol.), Cambridge: Cambridge University Press.

Quine Willard van Orman. (1969) 1971. Ontologische Relativität und andere Schriften, Stuttgart: Reclam.

Quine Willard van Orman. (1969) 1975. Replies To Chomsky. In Davidson D. and J. Hintikka (Hrsg.)., Words and Objections. Essays on the Work of W. V. Quine. Dordrecht revised edition.

Quine Willard van Orman. 1970. "On the Reason for the Indeterminacy of Translation”, Journal of Philosophy: 179-183. 
Quine Willard van Orman. (1974) 1994. Die Wurzeln der Referenz Frankfurt a. M. (Berlin): Suhrkamp Verlag.

Quine Willard van Orman. 1979. Word and Object. Cambridge Mass.: MIT Press

Quine Willard van Orman. 1990. Three Indeterminacies. In S.,R. B. Barrett, R. F. Gibson (Hrsg.)., Perspectives on Quine, 1-16. Cambridge: Blackwell.

Quine Willard van Orman, (1992) 1995. Unterwegs zur Wahrheit, Konzise Einleitung in die theoretische Philosophie, Paderborn: Ferdinand Schöning.

Quine Willard van Orman. 1995. From Stimulus zu Science, Cambridge Mas.: Harvard University Press.

Roth Michael. 1986. Bedeutungsverstehen und Sozialwissenschaft. Zu einem sozialen Rahmen der Einstellungszuschreibung, Frankfurt a. M.: Peter Lang Verlag.

Sartre Jean-Paul. (1936) 1964. Entwurf einer Theorie der Emotionen. In Die Transzendenz des Egos. Drei Essays, 153-195. Reinbeck bei Hamburg: Rowohlt Verlag.

Schantz Richard. 1996. Wahrheit, Referenz und Realismus. Eine Studie zur Sprachphilosophie und Metaphysik, Berlin: Walter de Gruyter.

Schnädelbach Herbert. 1971. Erfahrung, Begründung und Reflexion. Versuch über den Positivismus, Frankfurt a. M. (Berlin): Suhrkamp Verlag.

Stegmüller Wolfgang (Hrsg.). 1978. Das Universalien-Problem, Darmstadt: Wissenschaftliche Buchgesellschaft.

\author{
Abstract \\ From Radical Translation to Radical Interpretation - \\ Quine, Davidson and beyond
}

The Willard Van Orman Quine and Donald Davidson-Tradition was a dominant philosophy over thirty years beginning in the 1970s years in the theory of interpretation (language), epistemology and ontology. As my first step I give a sketch of Quine's turn from the theory of meaning to a theory of translation. This is at the same time a turn to a naturalized epistemology, the post-empiricism theory of meaning and to the radical translation and its basis theory. The post-empiricism theory of meaning states: beliefs and other attitudes and the meaning of sentences are connected inextricably. The naturalized epistemology claims to break the interplay between both by the stimulus meaning as a non-conceptual net-meaning. In the next step is argued that the stimulus meaning as the net meaning cannot explain the epistemic constraints of radical translation.

Subsequently to Quine's turn, I analyse Davidson's rebuilding of the radical translation into the radical interpretation, as well as his semantic model of radical 
translation as a continuation of the problem dimension, yet at the same time as a rejection of the basis theory of radical translation. It is of particular interest whether the truth-conditional theory of meaning and the application of the principle of charity (consistency and the truth of the beliefs of the speaker) as a constraint of every interpretation can close the epistemic gap. It is shown that the utterances of a speaker under study cannot be understood by the application of the principle of charity as the constraints of radical interpretation generally.

At the same time it should be considered whether a theory of truth and meaning in the style of A. Tarski gives us a satisfactory answer of the instantiation of the truthpredicate. Finally, I give a sketch of an alternative framework of interpretation.

Key words: Davidson, Quine, post-empiricism, radical translation, radical interpretation, Tarski, naturalized epistemology, meaning.

(Wykład wygłoszony na międzynarodowej konferencji Trans-Late - Über-Setzen - Tra-Duire. Language Diversity and Intercultural Hermeneutics Sprachdiversität und interkulturelle Hermeneutik. 15-17.05.2015) 